Submitted to ApJ January 13, 2015; AcCepted March 5, 2015

Preprint typeset using $\mathrm{LATE}_{\mathrm{E}} \mathrm{X}$ style emulateapj v. 05/12/14

\title{
DISCOVERY OF A YOUNG PLANETARY MASS COMPANION TO THE NEARBY M DWARF VHS J125601.92-125723.9*
}

\author{
Bartosz Gauza ${ }^{1,2}$, Victor J. S. BéJar ${ }^{1,2}$, Antonio Pérez-Garrido ${ }^{3}$, Maria Rosa Zapatero Osorio ${ }^{4}$, \\ Nicolas Lodieu ${ }^{1,2}$, Rafael Rebolo ${ }^{1,2,5}$, Enric Pallé ${ }^{1,2}$ and Grzegorz NowaK ${ }^{1,2}$ \\ Submitted to ApJ January 13, 2015; Accepted March 5, 2015
}

\begin{abstract}
In a search for common proper motion companions using the VISTA Hemisphere Survey (VHS) and the 2MASS catalogs we have identified a very red $\left(J-K_{s}=2.47 \mathrm{mag}\right)$ late-L dwarf companion of a previously unrecognized M dwarf VHS J125601.92-125723.9 (hereafter VHS 1256-1257), located at a projected angular separation of $8 . \prime 06 \pm 0$. . 03 . In this work we present a suite of astrometric, photometric, and spectroscopic observations of this new pair in an effort to confirm the companionship and characterize the components. From low-resolution $(R \sim 130-600)$ optical and near-infrared spectroscopy we classified the primary and the companion as an M7.5 \pm 0.5 and L7 \pm 1.5 , respectively. The primary shows slightly weaker alkali lines than field dwarfs of similar spectral type, but still consistent with either a high-gravity dwarf or a younger object of hundreds of millions of years. The secondary shows spectral features characteristic for low surface gravity objects at ages below several hundred million years, like the peaked triangular shape of the $H$-band continuum and alkali lines weaker than in field dwarfs of the same spectral type. The absence of lithium in the atmosphere of the primary and the likely kinematic membership to the Local Association allowed us to constrain the age of the system to the range of 150-300 Myr. We report a measurement of the trigonometric parallax $\pi=78.8 \pm 6.4$ mas, which translates into a distance of $12.7 \pm 1.0 \mathrm{pc}$; the pair thus has a projected physical separation of $102 \pm 9$ AU. We derived the bolometric luminosities of the components and compared them with theoretical evolutionary models to estimate the masses and effective temperatures. For the primary, we determined a luminosity of $\log \left(L_{\mathrm{bol}} / L_{\odot}\right)=-3.14 \pm 0.10$, and inferred a mass of $73_{-15}^{+20} M_{\mathrm{Jup}}$ at the boundary between stars and brown dwarfs and an effective temperature of $2620 \pm 140 \mathrm{~K}$. For the companion we obtained a luminosity of $\log \left(L_{\mathrm{bol}} / L_{\odot}\right)=-5.05 \pm 0.22$ and a mass of $11.2_{-1.8}^{+9.7} M_{\text {Jup }}$ placing it near the deuterium-burning mass limit. The effective temperature derived from evolutionary models is $880_{-110}^{+140} \mathrm{~K}$, about $400-700 \mathrm{~K}$ cooler than the temperature expected for field late-L dwarfs.

Subject headings: stars: brown dwarfs - stars: imaging - infrared: planetary systems - stars: individual
\end{abstract}

(VHS J125601.92-125723.9)

\section{INTRODUCTION}

Very low-mass stars and brown dwarfs directly imaged around stars constitute an important group for studies of the properties of substellar objects. We can infer the distance and metallicity of the companion from the brighter, more easily characterizable primary star. Most importantly, we can constrain the age of the system and thus overcome the intrinsic degeneracy between mass and age for the temperature and luminosity of objects below the hydrogen burning limit. This allows for a more thorough characterization and offers the opportunity to better understand their physical properties and to test evolutionary and atmospheric models (e.g., Pinfield et al. 2006.

${ }^{1}$ Instituto de Astrofísica de Canarias (IAC), Calle Vía Láctea s/n, E-38200 La Laguna, Tenerife, Spain

2 Departamento de Astrofísica, Universidad de La Laguna (ULL), E-38205 La Laguna, Tenerife, Spain

${ }^{3}$ Dpto. Física Aplicada, Universidad Politécnica de Cartagena, Campus Muralla del Mar, Cartagena, Murcia E-30202, Spain

${ }_{4}^{4}$ Centro de Astrobiología (CSIC-INTA), Ctra. Ajalvir km 4, 28850, Torrejón de Ardoz, Madrid, Spain

${ }^{5}$ Consejo Superior de Investigaciones Científicas, CSIC, Spain
}

Faherty et al. 2010). There are about 1500 objects spectrally classified as M7 and later, the vast majority is found to be isolated, single sources that appear to have spectral energy distributions, photometric colors, and kinematics consistent with a field population with ages in the range from 2 to 8 Gyr (Knapp et al. 2004; Cruz et al. 2007; Faherty et al. 2009). About 120 ultracool dwarfs (late-M, L, 'T, and Y dwarfs) have been confirmed as components of binary or multiple systems (Faherty et al. 2010, Deacon et al. 2014). These objects discovered over the last two decades have exhibited a large diversity in ages, atmospheric properties, and chemical compositions.

Surveys aimed at identifying low-mass companions of young stars (e.g., Wahhaj et al. 2011; Bowler et al. 2013, Chauvin et al. 2014), and the least massive members of young star clusters (e.g., Béjar et al. 2011; Peña Ramírez et al. 2012, Zapatero Osorio et al. 2014b), as well as searches for $\mathrm{L}$ and ' $\mathrm{T}$ ' dwarts in the field using large sky area surveys (Kirkpatrick et al. 2006, Cruz et al. 2009) have revealed a number of ultracool dwarfs that exhibit photometric and spectral properties different from typical late-type objects of the field population. Some of these peculiarities have been attributed to low surface gravities and cloudy atmospheres, occurring at the early stages of evolution, below several hundred million years 




$\mathrm{J}$
$\mathrm{H}$
$\mathrm{K}_{\mathrm{S}}$

FIG. 1. - VISTA $Y J H K_{s}$ band images of the new common proper motion pair VHS 1256-1257. The identified companion is located at a position angle of $218.1 \pm 0.2$, with a projected angular separation of $8^{\prime \prime} .06 \pm 00^{\prime \prime} 03$ which corresponds to $\sim 102$ AU at the determined parallactic distance of $12.7 \mathrm{pc}$. The field of view is $30^{\prime \prime} \times 30^{\prime \prime}$, with north up and east to the left.

(Cruz et al. 2009; Allers \& Liu 2013; Zapatero Osorio et al. 2014a). Young L dwarfs, either free-floating or as companions to stars, were found to share similar characteristics. They have very red colors $\left(J-K_{s}>2 \mathrm{mag}\right)$, $J$-band absolute magnitudes fainter than their old, field counterparts, and show distinctive spectral features such as, for example, a sharply peaked, triangal-shaped continuum in the $H$ band and weaker sodium and potassium lines.

Recent studies have revealed a strong resemblance between the young L dwarfs and directly imaged planetary mass companions (e.g., 2MASS 1207-39 b, Chauvin et al. 2005, HR 8799 bcde, Marois et al.|2008, 2010, GJ 504 b Kuzuhara et al. 2013, $\beta$ Pic b, Lagrange et al. 2009). They have similar near-infrared (near-IR) colors and absolute magnitudes, overlapping effective temperature regimes of $\sim 1000-1500 \mathrm{~K}$ and masses of a few to a few tens of Jupiter masses (Barman et al. 2011a; Bowler

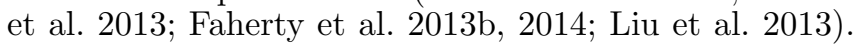
Spectroscopic and photometric studies of young substellar objects can provide information on the physical properties of gas giant exoplanets found by transit and radial velocity surveys, in particular, on the characteristics and composition of their complex atmospheres.

In this work we present the identification and characterization of a nearby, young binary system with components that we classify as M7.5 \pm 0.5 and L7 \pm 1.5 . In Section 2 we describe the data and search method that led to the identification of the pair. Section 3 contains the description of observations aimed at confirming their companionship and characterization. In Section 4 we discuss their physical properties. We classify their spectral types, and determine the distance and radial and space velocities. We constrain the possible age of the system and estimate the luminosities, masses, and effective temperatures of the two components. Final remarks and conclusions are presented in Section 5.

\section{IDENTIFICATION OF THE SYSTEM}

\subsection{VISTA Hemisphere Survey Data}

The reported common proper motion pair was identified using the VISTA Hemisphere Survey (VHS) data and the 2MASS (Skrutskie et al. 2006). The VHS is a near-IR ESO public survey designed to map the entire southern hemisphere in the $J$ and $K_{s}$ broad-band filters with average $5 \sigma$ depths of $J=20.2 \mathrm{mag}$ and $K_{s}=18.1 \mathrm{mag}$ (McMahon et al. 2013). In some particular areas also observations in the $Y$ and $H$ bands are also performed.
The 4-m VISTA telescope (Emerson 2001; Emerson et al. 2004) has operated since 2009 at ESO's Cerro Paranal Observatory in Chile and has thus far covered about 8000 $\operatorname{deg}^{2}$ of the sky. It is equipped with a wide-field infrared camera VIRCAM (Dalton et al. 2006) composed of 16 Raytheon detectors $2048 \times 2048$ pixel array each, with a mean plate scale of 0.34 , giving a field of view of $1.65^{\circ}$ in diameter.

The VHS images are processed and calibrated automatically by a dedicated science pipeline implemented by the Cambridge Astronomical Survey Unit (CASU). Standard reduction and processing steps include dark and sky subtraction, flat-field correction, linearity correction, destripe, and jitter stacking. For a detailed description we refer the reader to the CASU webpage http://casu. ast.cam.ac.uk/surveys-projects/vista as well as to Irwin et al. (2004) and Lewis et al. (2010).

The photometry provided in the VHS catalog is calibrated using the 2MASS magnitudes of all matching stars converted onto the VISTA system applying color equations ${ }^{6}$, which include terms accounting for the interstellar reddening. Photometric calibrations are determined to an accuracy of $1 \%-2 \%$. The astrometric solution for VHS observations is obtained through the CASU pipeline, using the 2MASS point source catalog. The objects on the catalogs extracted from each detector are matched to their counterparts in 2MASS using a correlation radius of $1^{\prime \prime}$. Because 2MASS has a high degree of internal consistency it is possible to calibrate the world coordinate system of VISTA images to relative accuracy better than 0 ! 1 .

\subsection{Search Method}

Using VHS data, we carried out a search for high proper motion objects, by cross-matching VHS sources with the 2MASS Point Source Catalog (Skrutskie et al. 2006). The search focused on objects that had moved at least $2^{\prime \prime}$ and a maximum of $30^{\prime \prime}$ from 2 MASS to VHS. The time baseline between the two surveys is typically about $12 \mathrm{yr}$, which gives proper motions of approximately 0.15 $3.0 \operatorname{arcsec}_{\mathrm{yr}^{-1}}$. Over the common area of $\sim 5000 \mathrm{deg}^{2}$ between 2MASS and VHS we found more than 6000 objects with $J$ magnitudes in the range $11-17 \mathrm{mag}$ and


correlated this catalog with WISE (Wright et al. 2010), so that near and mid-infrared information is available for

6 http://casu.ast.cam.ac.uk/surveys-projects/vista/ technical/photometric-properties 
each target. Most of the high proper motion objects are relatively nearby $\mathrm{M}$ dwarfs with estimated photometric distances $<50 \mathrm{pc}$ (A. Perez-Garrido et al., in preparation). We have searched for common proper motion pairs and multiples among these objects with proper motion consistent within 40 mas yr$^{-1}$ in both right ascension and declination $\left(\mu_{\alpha}, \mu_{\delta}\right)$. VHS 1256-1257 system was one of the identified candidates. The pair was observed with the VISTA $Y J H K_{s}$ filters and with a seeing of 1"'1 on 2011 July 1 . The primary and the secondary were cataloged in 2MASS with designations 2MASS J125602.15125721.7 and 2MASS J125601.83-125727.6, respectively. The primary was also listed as SIPS 1256-1257 in the sample of low-mass stars with $\mu>00^{\prime \prime} 1 \mathrm{yr}^{-1}$ from Deacon \& Hambly (2007), with measured proper motion amplitude $\mu=0^{\prime \prime} 357 \mathrm{yr}^{-1}$ and the position angle of the proper motion vector of $244.76^{\circ}$.

VISTA $Y J H K_{s}$ images of the pair are presented in Fig.1 and the photometry is given in Table 2 The companion is located at a projected angular separation of $88^{\prime \prime} 06 \pm 0 . \prime 03$, at a position angle of $218.1 \pm 0.2$. This separation corresponds to a projected orbital separation of $102 \pm 9 \mathrm{AU}$ at the estimated distance of the system (Section 4.2). The two components share a common proper motion, which significantly differs from the proper motion of background stars as shown in Fig. 2. The $\mu_{\alpha} \cos \delta$ and $\mu_{\delta}$ measured from the VHS and 2MASS positions of the sources, were $-270 \pm 17,-185 \pm 14$ and $-292 \pm 27$ and $-212 \pm 23$ mas $\mathrm{yr}^{-1}$, for the primary and secondary, respectively. The uncertainties in proper motion of each component correspond to the rms of $\mu_{\alpha} \cos \delta$ and $\mu_{\delta}$ of the background stars within a one-degree radius around the primary, and magnitudes similar to that of the given component. Time baseline between the two epochs is 12.3 yr. The primary has $J=11.02 \pm 0.02 \mathrm{mag}$ and $J-K_{s}=0.97 \mathrm{mag}$ (2MASS photometry, VIRCAM is out of the linear range) The secondary is roughly six magnitudes fainter in the $J$ band $(J=17.14 \pm 0.02 \mathrm{mag})$ and has a very red $J-K_{s}$ color of 2.47 mag indicating its significantly cooler type and lower mass with respect to the primary.

\section{FOLLOW-UP OBSERVATIONS AND DATA REDUCTION}

\subsection{NTT/SofI Near-infrared Spectroscopy and Imaging}

To measure the infrared spectral types of the VHS 1256-1257 components we performed follow-up near-IR spectroscopy using the Son of ISAAC (SofI) spectro-imager installed on the $3.6 \mathrm{~m}$ New Technology Telescope (NTT) on 2014 March 12. SofI is equipped with a Hawaii $\mathrm{HgCdTe}$ detector with $1024 \times 1024$ pixel array offering a field of view of $4.9 \times 4.9 \operatorname{arcmin}^{2}$ with a 0 '.288 pixel scale. We have used blue and red grisms covering the $950-1640$ and $1530-2520 \mathrm{~nm}$ range, combined with a slit of $1^{\prime \prime}$, orientated along the position angle of the components of the system. This instrumental configuration provides a nominal dispersion of 6.96 and $10.22 \AA \mathrm{pix}^{-1}$ for the Blue and Red grating, respectively. To subtract the sky background we used an ABBA nodding pattern with an offset of $20^{\prime \prime}$ between the two positions. Individual exposure times were 600 and $900 \mathrm{~s}$ for the red and blue grism, respectively. Right af-

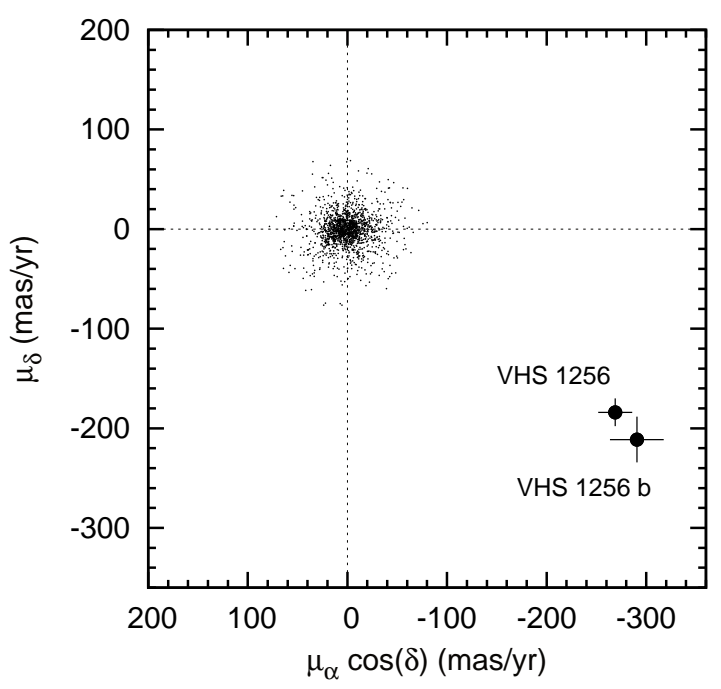

FIG. 2.- Proper motion diagram for the VHS 1256-1257 system. All correlated objects within one degree around the primary with $J<17.5$ mag are plotted with black dots. Components of the common proper motion pair are labeled. Error bars correspond to the astrometric RMS of stars near the magnitudes of the components.

ter VHS 1256-1257 we observed a telluric standard star HD $112304(V=6.19 \mathrm{mag}, \mathrm{A} 0 \mathrm{~V}, \mathrm{H} \varnothing \mathrm{g}$ et al. 2000 $\operatorname{van}$ Leeuwen 2007). The sky conditions during the observations were clear with sub-arcsecond seeing.

The two-dimensional (2D) images were reduced and combined using the ESO SofI pipeline run within the Gasgano environment. The traces of the primary and the companion spectra on the 2D images were clearly separated and there was no contamination between the two sources. The two spectra of the VHS 1256-1257 system and of HD 112304 were then extracted using standard IRAF routines within the APALL package and wavelength calibrated via Xenon arc lines. The dispersion solution had an rms of 0.4 and $0.35 \AA$ for the blue and red part of the spectrum, respectively. The final resolution of the spectrum was $24 \AA(R \sim 600)$. Telluric absorption lines were corrected, dividing the spectra by the A0V telluric standard HD 112304 observed at a similar airmass, and multiplying by a blackbody of a corresponding effective temperature of $9480 \mathrm{~K}$. The obtained NTT SofI spectra of VHS 1256-1257 primary and companion normalized at $1.6 \mu \mathrm{m}$ are displayed in Figure 3 .

On 2014 April 27 we used SofI in imaging mode, to perform $J$ - and $H$-band observations at an additional epoch. We used a nine-position dither pattern with individual exposures of $5 \mathrm{~s}$. The weather was clear, with a seeing of $11^{\prime \prime} 15-1{ }^{\prime \prime} 30$. Raw images were reduced using the ESO SofI Pipeline within Gasgano, which includes bias subtraction, flat-field correction, plane re-centering, and co-addition of individual frames to a final image. Images were employed for determining the trigonometric parallax of the system and for performing relative astrometry between the two components as described in Section 4.2. Table 4 provides these measurements.

\subsection{GTC/OSIRIS Optical Spectroscopy}

We obtained low-resolution optical spectroscopy of VHS 1256-1257 with the Optical System for Imag- 
TABLE 1

OBSERVATION LOG OF VHS 1256-1257.

\begin{tabular}{|c|c|c|c|c|c|c|c|c|c|}
\hline $\begin{array}{l}\text { Obs. Date } \\
\text { (UT) }\end{array}$ & "Tel/Instrument & Mode & $\begin{array}{c}\text { Wavelength } \\
(\mu \mathrm{m})\end{array}$ & $\begin{array}{c}\text { Exp. Time } \\
(\mathrm{s})\end{array}$ & $\begin{array}{c}\text { Seeing } \\
\left({ }^{\prime \prime}\right)\end{array}$ & $\begin{array}{l}\text { Slit } \\
\left({ }^{\prime \prime}\right)\end{array}$ & Grating & $\begin{array}{c}\text { Scale } \\
\left({ }^{\prime \prime} / \text { pix }\right)\end{array}$ & $\begin{array}{c}\text { Res. } \\
\text { Power }\end{array}$ \\
\hline $2011 \mathrm{Jul} 01^{\mathrm{a}}$ & VISTA/VIRCAM & $\operatorname{Img}$ & $Y J H K_{s}$ & $15,15,7.5,7.5$ & 1.0 & - & - & 0.34 & - \\
\hline $2014 \mathrm{Mar}_{02}{ }^{\mathrm{b}}$ & GTC/OSIRIS & Spec & $0.48-1.00$ & 120 & 0.57 & 1.5 & R500R & 0.25 & 320 \\
\hline $2014 \operatorname{Mar} 12^{\mathrm{a}}$ & NTT/SofI & Spec & $0.95-1.64$ & $4 \times 600$ & 0.8 & 1.0 & Blue & 0.29 & 600 \\
\hline $2014 \operatorname{Mar} 12^{\mathrm{a}}$ & NTT/SofI & Spec & $1.53-2.52$ & $4 \times 900$ & 0.8 & 1.0 & Red & 0.29 & 600 \\
\hline 2014 Apr 22, May 04 & NOT/ALFOSC & Spec & $0.63-0.68$ & $900,4 \times 900$ & $0.8-1.1$ & 1.3 & Grism\#17 & 0.19 & 4700 \\
\hline $2014 \operatorname{Apr} 27^{\mathrm{a}}$ & NTT/SofI & $\operatorname{Img}$ & $J H$ & $9 \times 2$ & $1.0-1.3$ & - & - & 0.29 & - \\
\hline 2014 May $14^{\mathrm{b}}$ & GTC/OSIRIS & Spec & $0.56-0.77$ & $3 \times 600$ & 0.56 & 0.6 & $\mathrm{R} 2500 \mathrm{R}$ & 0.25 & 3000 \\
\hline 2014 Jun $03^{\mathrm{a}}$ & GTC/OSIRIS & Spec & $0.48-1.00$ & $3 \times 600$ & 0.63 & 1.5 & R300R & 0.25 & 130 \\
\hline $2014 \mathrm{Jul} 01,09,10^{\mathrm{b}}$ & VLT/UVES & Spec & $0.37-0.49$ & $3 \times 3300$ & $0.5-1.7$ & 1.0 & $\mathrm{CD} 2,4$ & 0.22 & 40000 \\
\hline $2014 \mathrm{Jul} 01,09,10^{\mathrm{b}}$ & VLT/UVES & Spec & $0.56-0.95$ & $3 \times 3300$ & $0.5-1.7$ & 1.0 & $\mathrm{CD} 2,4$ & 0.16 & 40000 \\
\hline $2014 \mathrm{Jul} 15^{\mathrm{b}}$ & IAC80/CAMELOT & Img & $V I$ & 300 & 1.1 & - & - & 0.30 & - \\
\hline $2014 \mathrm{Jul} 17^{\mathrm{a}}$ & WHT/ACAM & $\operatorname{Img}$ & Sloan $i, z$ & $9 \times 30,9 \times 30$ & 0.85 & - & - & 0.25 & - \\
\hline $2014 \mathrm{Jul} 18^{\mathrm{a}}$ & WHT/LIRIS & $\operatorname{Img}$ & $J$ & $5 \times 9 \times 2$ & 0.7 & - & - & 0.25 & - \\
\hline 2014 Dec $19,20^{\mathrm{b}}$ & IAC80/CAMELOT & $\operatorname{Img}$ & $I$ & $6 \times 300$ & $1.5,1.2$ & - & - & 0.30 & - \\
\hline
\end{tabular}

a Both components were observed

b Only the primary was observed

ing and low-intermediate Resolution Integrated Spectroscopy (OSIRIS; Cepa 2010) mounted on the 10.4$\mathrm{m}$ Gran Telescopio de Canarias (GTC) telescope in La Palma. OSIRIS is equipped with two $2048 \times 4096$ Marconi CCD42-82 detectors offering a field of view approximately $7 \times 7 \operatorname{arcmin}^{2}$ with an unbinned pixel scale of 0 ". 125 . We observed the primary star using two different gratings: R500R, which allowed us to measure the general spectral energy distribution at a resolution of $R \sim 320$, covering the $0.48-1.00 \mu \mathrm{m}$ range, and $\mathrm{R} 2500 \mathrm{R}$ providing a resolution of $R \sim 3000$ in the $0.56-0.77 \mu \mathrm{m}$ region, which we used to study the $\mathrm{H} \alpha$ and $\mathrm{Li}$ I lines. Observations with R500R and R2500R were acquired on 2014 March 2 and May 14, respectively. On 2014 June 3 , both the primary and secondary were aligned on the 1.' 5 -width slit and were observed simultaneously using the R300R grating. This instrumental setup provided optical spectra with a resolving power of $R \sim 130$, which is optimal to maximize the signal-to-noise ratio of the faint companion data. Observations were performed in service mode (Table 1) as part of a GTC filler program (number GTC5-14A; PI Lodieu). The nights were dark with a clear sky and a seeing of $\sim 00^{\prime \prime} 6$. Details of the used instrumental configurations and exposure times are provided in Table 1. The spectrophotometric standards, Hiltner 600, GD 153, and Ross 640 (Høg et al. 1998, 2000 Pancino et al. 2012) were observed with the R500R+GR, R2500R, and R300R gratings, respectively, on the same night of the scientific observations. Observations of the standards with R300R and R500R were done with the same grating combined with the Sloan $z$ filter to correct for second-order contamination beyond $9200 \AA$ (see procedure in Zapatero Osorio et al. 2014a). Bias frames, continuum lamp flat fields, and Neon + Xenon arc lamps were observed by the observatory staff during the afternoon preceding the observations.

We reduced the OSIRIS spectra using routines within IRAF. We subtracted the raw spectra by a mediancombined bias and divided by a normalized continuum lamp flat field. From the optimally combined 2D images we extracted the spectra using the APALL routine and calibrated in wavelength with the lines from the combined arc lamp. To improve the detection of the secondary in observations with R300R we inserted both components in the slit and used the trace of the primary as a reference to combine the individual exposures. Correction of the instrumental response was done using the corresponding spectrophotometric standards observed during the same nights. The obtained spectra are displayed in Figures 3 . 4 and 6 .

\subsection{NOT/ALFOSC Optical Spectroscopy}

For a first estimate of the radial velocity of the primary star, intermediate-resolution optical spectroscopy was acquired for VHS 1256-1257 A using the Andalucia Faint Object Spectrograph and Camera (ALFOSC) instrument of the $2.5 \mathrm{~m}$ Nordic Optical Telescope (NOT) operating at the Observatorio del Roque de los Muchachos, La Palma. The camera uses a E2V back illuminated CCD 42-40 chip with $2 \mathrm{k} \times 2 \mathrm{k}$ pixels with a scale of 0.19 $\operatorname{arcsec~pix~}^{-1}$, providing a field of view of $6.4 \times 6.4 \mathrm{arcmin}^{2}$. We observed the primary in two different epochs, on the nights of 2014 April 22 and May 5. For the instrument setup we chose Grism\#17 and a 1". 3 slit providing a wavelength range of $6330.4-6853.6 \AA$ and a nominal resolution of $0.255 \AA \mathrm{pix}^{-1}$. On the first night one useful exposure of $900 \mathrm{~s}$ was obtained under the presence of variable clouds. On the second night four exposures of $900 \mathrm{~s}$ were taken, under clear sky conditions. The seeing was similar on both nights $\left(0 .{ }^{\prime \prime}-1^{\prime \prime} 1\right)$, and hence the final resolution of the spectra was also very similar: $1.5 \AA(R \sim 4700)$. During the same nights using the same instrumental configuration, we also observed the star GJ 388, which is a $\mathrm{M} 4.5 \mathrm{~V}$ with a precise radial velocity determination of $v_{r}=12.453 \pm 0.066 \mathrm{~km} \mathrm{~s}^{-1}$ by Chubak et al. (2012).

Raw data were reduced using routines within the IRAF environment. Two dimension images were bias corrected and flat fielded using continuum lamps normalized using the RESPONSE routine. Spectra were optimally extracted using the APALL routine and were wavelength calibrated using HeNe lamps and a cubic spline function fit of the order of three, providing an rms better than $0.06 \AA$. Final spectra were corrected by the instrumental response using the spectrophotometric standard HZ44.

\subsection{VLT/UVES Optical Spectroscopy}

To measure a more precise radial velocity and to investigate in detail the spectral signatures of youth of the pri- 
mary star, like the Li I line at $670.82 \mathrm{~nm}$ we also obtained a high-resolution spectrum of VHS 1256-1257 A using the Ultraviolet and Visual Echelle Spectrograph (UVES) mounted on the Kueyen unit of the ESO Very Large Telescope. UVES is a two-arm crossdispersed echelle spectrograph covering the wavelength range $300-500 \mathrm{~nm}$ (blue) and 420-1100 $\mathrm{nm}$ (red), with the possibility to use dichroic beam splitters. The instrument is equipped with a single chip in the blue arm and a mosaic of two chips in the red arm. The blue CCD is a $2 \mathrm{~K} \times 4 \mathrm{~K}, 15 \mu \mathrm{m}$ pixel size thinned EEV CCD-44. The red CCD mosaic is made of an EEV chip of the same type and the MIT/LL CCID-20 chip. Each arm has two cross disperser gratings working in first spectral order; the typical order of separation is $10^{\prime \prime}$. We have used the standard dichroic mode setting, which covers the $565-950 \mathrm{~nm}$ spectral range in the red arm, with the central wavelength at $760 \mathrm{~nm}$, and a $373-499 \mathrm{~nm}$ range in the blue arm, centered at $437 \mathrm{~nm}$. This configuration, with 1 arcsec slit, provides spectral resolution of $R \sim 40000(25 \mathrm{~mA} / \mathrm{pix})$. Observations were performed in service mode under the ESO DDT program 293.C-5014(A) on 2014 July 1, 9 and 10, with clear sky conditions. The total integration time was $9900 \mathrm{~s}$ divided into three exposures of $3300 \mathrm{~s}$.

The spectra were reduced, extracted, and calibrated using the ESO UVES pipelines under the ESO Recipe Flexible Execution Workbench environment (Reflex, Freudling et al. 2013). The reduction steps of the workflow executed by the software include creation of master flat and bias frames, with the corresponding corrections of science data and detection of the order positions on the detector. Subsequently, a wavelength calibration solution is obtained from an input arc-lamp frames. We have used the ThAr arcs acquired in both blue and red arms, during the afternoon preceding the observations. Instrument characteristics description provides a note of caution in that in the spectral region above $700 \mathrm{~nm}$ the ThAr lamp has some very bright Argon lines that saturate the CCDs, and the heavily saturated lines may contain remnants in the following exposures. Here the calibration frames were taken more than four hours before the night, i.e. more than the typical time after which the remnants vanish. Then, the spectrum from each order is extracted and merged. Last, the flux-calibration of the science spectrum is carried out, using the appropriate instrument response curve, in the blue part obtained from a standard star observation, and from the instrument master response curve in the red part. We have corrected the telluric absorption features in the spectrum using the ESO Molecfit software (Smette et al. 2015, Kausch et al. 2015). The results obtained using the UVES data are described in Section 4.4, with the final spectra presented in Figures 10 and 11.

\subsection{IAC80/CAMELOT Imaging}

On 2014 July 15 and December 19 and 20 we performed imaging observations of the primary using CCD Camera CAMELOT of the $0.8 \mathrm{~m}$ IAC80 telescope at the Teide Observatory on Tenerife. We aimed to obtain optical photometry in the VI (Johnson-Bessell's) filters. The images were also used in the parallax determination. The camera contains a E2V $2048 \times 2048$ back illuminated chip with 0 !' 304 pixels corresponding to a $10.4 \times 10.4$ arcmin $^{2}$ field of view. Individual exposures of $300 \mathrm{~s}$ were obtained
TABLE 2

MEASUREMENTS AND DETERMINED PHYSICAL PARAMETERS OF VHS 1256-1257 SYSTEM

\begin{tabular}{|c|c|c|}
\hline Astrometry & Primary & Companion \\
\hline R.A. $(J 2000)^{a}$ & $12^{\mathrm{h}} 56^{\mathrm{m}} 01^{\mathrm{s}} .922$ & $12^{\mathrm{h}} 56^{\mathrm{m}} 01^{\mathrm{s}} .586$ \\
\hline Decl. $(\mathrm{J} 2000)^{\mathrm{a}}$ & $-12^{\circ} 57^{\prime} 23^{\prime \prime} \cdot 990$ & $-12^{\circ} 57^{\prime} 30^{\prime \prime} 310$ \\
\hline 2MASS ID & J125602.15-125721.7 & J125601.83-125727.6 \\
\hline Separation $(\operatorname{arcsec})^{\mathrm{a}}$ & \multicolumn{2}{|c|}{$8.06 \pm 0.03$} \\
\hline Separation (AU) & \multicolumn{2}{|c|}{$102 \pm 9$} \\
\hline Position angle $(\mathrm{deg})^{\mathrm{a}}$ & \multicolumn{2}{|c|}{$218.1 \pm 0.2$} \\
\hline$\mu_{\alpha} \cos \delta\left(\operatorname{mas~yr}^{-1}\right)$ & $-281.5 \pm 5.3$ & $-275.4 \pm 5.3$ \\
\hline$\mu_{\delta}\left(\operatorname{mas~yr}^{-1}\right)$ & $-205.5 \pm 15.2$ & $-198.4 \pm 15.2$ \\
\hline Parallax $\pi$ (mas) & \multicolumn{2}{|c|}{$78.8 \pm 6.4$} \\
\hline Distance $d(\mathrm{pc})$ & \multicolumn{2}{|c|}{$12.7 \pm 1.0$} \\
\hline$v_{r}\left(\mathrm{~km} \mathrm{~s}^{-1}\right)$ & $-1.4 \pm 4.5$ & $\ldots$ \\
\hline$U\left(\mathrm{~km} \mathrm{~s}^{-1}\right)$ & $-9.4 \pm 2.0$ & $\ldots$ \\
\hline$V\left(\mathrm{~km} \mathrm{~s}^{-1}\right)$ & $-16.4 \pm 3.0$ & $\ldots$ \\
\hline$W\left(\mathrm{~km} \mathrm{~s}^{-1}\right)$ & $-8.5 \pm 3.9$ & $\ldots$ \\
\hline \multicolumn{3}{|l|}{ Photometry } \\
\hline$V$ (mag) & $17.759 \pm 0.059$ & $\ldots$ \\
\hline$I$ (mag) & $13.318 \pm 0.039$ & $\ldots$ \\
\hline Sloan $i$ (mag) & $\ldots$ & $22.494 \pm 0.315$ \\
\hline Sloan $z$ (mag) & . & $20.095 \pm 0.090$ \\
\hline 2MASS $J$ (mag) & $11.018 \pm 0.023$ & $16.662 \pm 0.287$ \\
\hline 2MASS $H$ (mag) & $10.473 \pm 0.023$ & $15.595 \pm 0.209$ \\
\hline 2MASS $K_{s}(\mathrm{mag})$ & $10.044 \pm 0.021$ & $14.568 \pm 0.121$ \\
\hline VHS $Y$ (mag) & $<11.72^{\mathrm{b}}$ & $18.558 \pm 0.051$ \\
\hline VHS $J$ (mag) & $<11.36^{\mathrm{b}}$ & $17.136 \pm 0.020$ \\
\hline VHS $H$ (mag) & $<11.02^{\mathrm{b}}$ & $15.777 \pm 0.015$ \\
\hline VHS $K_{s}$ (mag) & $<10.42^{\mathrm{b}}$ & $14.665 \pm 0.010$ \\
\hline WISE W1 (mag) & $9.880 \pm 0.023$ & $13.6 \pm 0.5$ \\
\hline WISE W2 (mag) & $9.658 \pm 0.021$ & $12.8 \pm 0.5$ \\
\hline WISE W3 (mag) & $9.390 \pm 0.044$ & $>11.8$ \\
\hline WISE W4 (mag) & $8.334 \pm 0.410$ & $>8.65$ \\
\hline \multicolumn{3}{|l|}{ Spectral Classification } \\
\hline Optical & $\mathrm{M} 7.0 \pm 0.5$ & $\mathrm{~L} 8.0 \pm 2.0$ \\
\hline Near-IR $J$ & $\mathrm{M} 8.0 \pm 0.5$ & $\mathrm{~L} 8.0 \pm 1.0$ \\
\hline Near-IR $K$ & $\mathrm{M} 8.0 \pm 0.5$ & $\mathrm{~L} 5.0 \pm 2.0$ \\
\hline $\mathrm{H}_{2} \mathrm{O}$ index & $\mathrm{M} 7.6 \pm 0.4$ & $>\mathrm{L} 4.0$ \\
\hline $\mathrm{H}_{2} \mathrm{OD}$ index & $<\mathrm{L} 0$ & $\mathrm{~L} 5.8 \pm 0.8$ \\
\hline $\mathrm{H}_{2} \mathrm{O}-1$ index & $\mathrm{M} 6.9 \pm 1.1$ & $\ldots$ \\
\hline $\mathrm{H}_{2} \mathrm{O}-2$ index & $\mathrm{M} 7.9 \pm 0.5$ & $\ldots$ \\
\hline Adopted spectral type & $\mathrm{M} 7.5 \pm 0.5$ & $\mathrm{~L} 7.0 \pm 1.5$ \\
\hline Near-IR gravity class & INT-G & VL-G \\
\hline \multicolumn{3}{|l|}{ Physical Properties } \\
\hline Age (Myr) & \multicolumn{2}{|c|}{$150-300$} \\
\hline $\log \left(L_{\mathrm{bol}} / L_{\odot}\right)$ & $-3.14 \pm 0.10$ & $-5.05 \pm 0.22$ \\
\hline $\operatorname{Mass}\left(M_{\mathrm{Jup}}\right)$ & $73_{-15}^{+20}$ & $11.2_{-1.8}^{+9.7}$ \\
\hline$T_{\text {eff }}(\mathrm{K})$ & $2620 \pm 140$ & $880_{-110}^{+140}$ \\
\hline $\log (g)$ & $5.05 \pm 0.10$ & $4.25_{-0.10}^{+0.35}$ \\
\hline
\end{tabular}

${ }^{\text {a }}$ Measured using VHS images, epoch (MJD) $=55743.067635$

b $Y J H K_{s}$ photometry out of VIRCAM linear range

in the $V$ and $I$ filters. On the night of July 15, right after the scientific target, we observed two standard star fields at similar airmass, containing eight standard stars used for the photometry calibration. Weather conditions were clear/photometric with seeing of 1 .'1-1."3.

The data were reduced automatically using a dedicated PyRAF script that includes a standard bias and flat-field corrections procedure. We carried out aperture photometry with the PHOT task within the IRAF environment. Photometric calibration of the instrumental magnitudes in each filter was obtained using eight standard stars (Landolt 1992, 2009) from the two observed fields. The uncertainty of the calibration is $\sim 0.04 \mathrm{mag}$. 


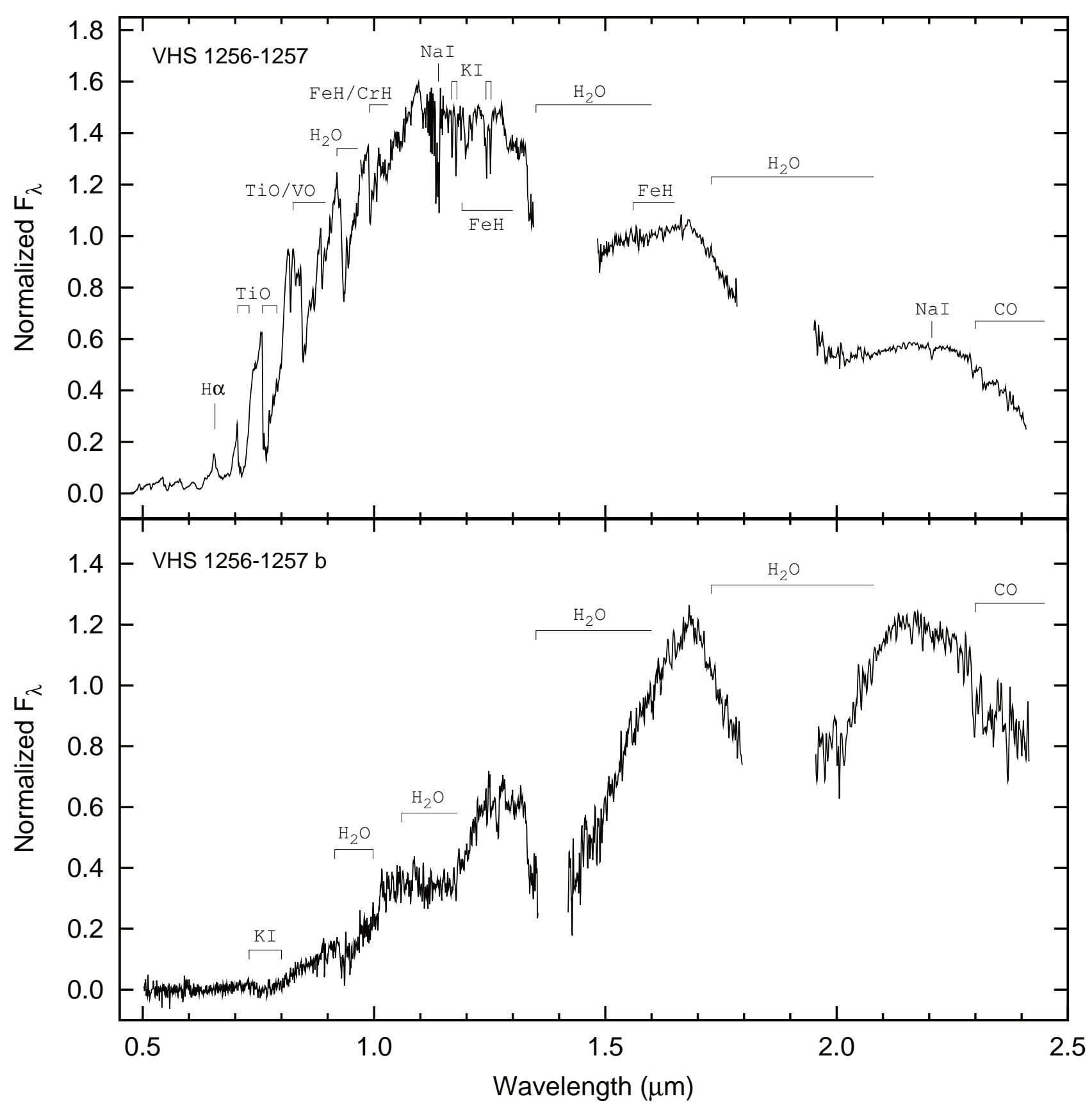

FIG. 3.- Low-resolution optical $(0.5-1.0 \mu \mathrm{m}, R \sim 120-320)$ and near-infrared (1.0-2.4 $\mu \mathrm{m}, R \sim 600)$ spectra of the primary (top) and companion (bottom) obtained using GTC/OSIRIS and NTT/SofI. The flux is normalized at $1.6 \mu \mathrm{m}$. The most prominent molecular and atomic features are indicated. Unlike most of old population field L dwarfs, the spectrum of VHS 1256-1257 b peaks in the $H$ band, which has a triangle-shaped continuum characteristic for young, dusty L dwarfs.

The measured VI magnitudes of the primary and other photometric data of the primary and the companion are listed in Table 2

\subsection{WHT/ACAM and LIRIS Imaging}

To complement the photometric information at different wavelengths, we also acquired optical images of the pair using the Sloan $i$ and $z$ broad-band filters. The final $z$-band image was also employed to constrain the trigonometric parallax (Section 4.2.2). Observations were done using the ACAM camera at the 4.2-m William Herschel
Telescope (WHT) on 2014 July 17. ACAM uses a $2 \mathrm{k} \times 2 \mathrm{k}$ pixels EEV CCD detector with $0.25 \operatorname{arcsec} \mathrm{pix}^{-1}$ scale, providing a circular field of view with a $8.3 \mathrm{arcmin}^{2}$ diameter. We used a nine-point dither pattern with individual exposures of $30 \mathrm{~s}$. On the same observing run on July 18 we have obtained $J$-band observations using the Long-slit Intermediate Resolution Infrared Spectrograph (LIRIS) spectrograph and imager on the WHT, for an additional astrometry epoch of the binary used to constrain the parallax measurement. LIRIS uses a $1 \mathrm{k} \times 1 \mathrm{k}$ 

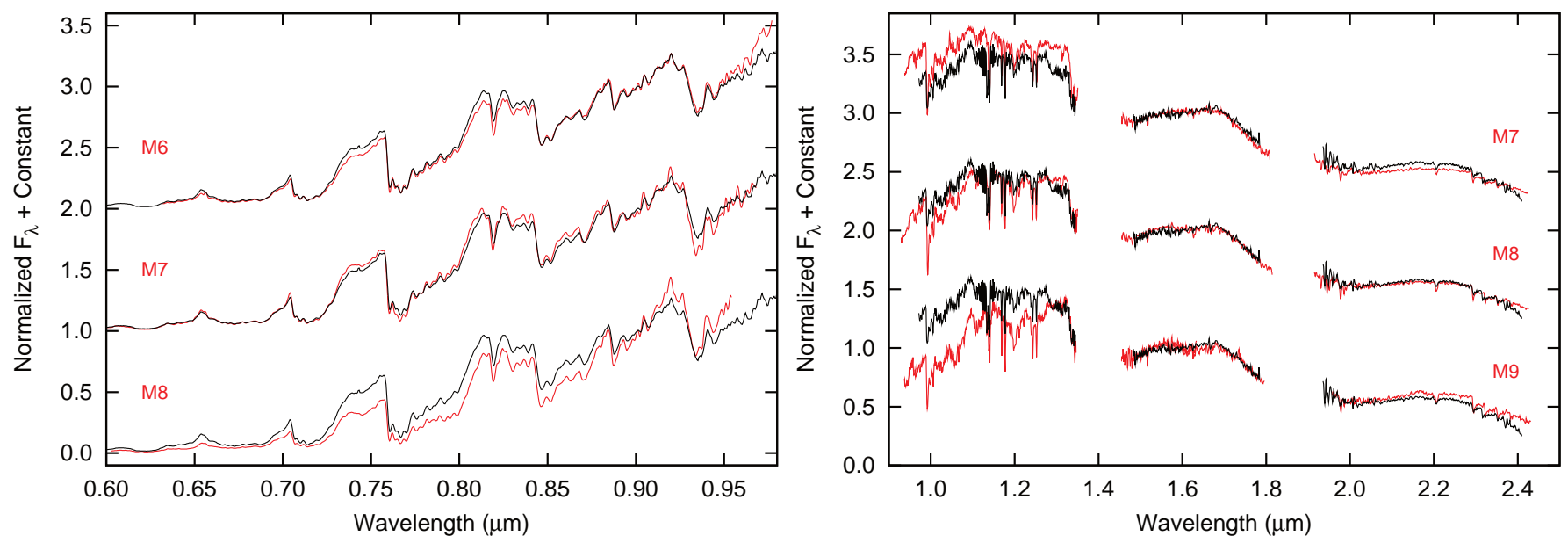

FIG. 4.- Low-resolution GTC/OSIRIS optical (left) and NTT/SofI near-infrared (right) spectra of the primary VHS 1256-1257 plotted with black line, compared with late-M spectral templates (with labels indicating their type). Comparison spectra were smoothed to match the resolution of our data. The sources and references of used templates are described in Section 4.1.1. Spectra were normalized at 0.9 $\mu \mathrm{m}$ at optical and at $1.6 \mu \mathrm{m}$ at near-IR and offset by a constant for display.

HAWAII detector with a pixel scale of $0.25 \operatorname{arcsec} \mathrm{pix}^{-1}$, yielding a field of view of $4.27 \times 4.27 \mathrm{arcmin}^{2}$. We used a sequence of five dither patterns of 9 positions and $2 \mathrm{~s}$ individual exposures, giving a total exposure time of $90 \mathrm{~s}$. Weather conditions were clear during the observations, with average seeing of $00^{\prime \prime} 85$ on the first and $0{ }^{\prime \prime} 66-0{ }^{\prime \prime} 68$ on the second night.

We carried out the data reduction of the LIRIS images with the LIRIS data reduction packag $\AA^{7}$. The procedure consisted of subtracting the sky made from the dithered images, correction for flat field, vertical gradient observed on the detector and the geometrical distortion. ACAM images were reduced with standard procedures using IRAF. Master sky flat frames were obtained from science images by combining by the median the individual exposures of the dither sequence. Corrected images were aligned and average combined. The aperture photometry of the companion was obtained using the PHOT task within IRAF. To calibrate the instrumental magnitudes we measured the photometry of stars in a field observed after VHS 1256-1257, under similar weather and airmass conditions. We selected 10 point sources with a good signal-to-noise ratio and available measurement in the SDSS Photometric Catalog (Ahn et al. 2012). The obtained $i$ and $z$ magnitudes are listed in Table 2, The primary was saturated in the individual exposures.

\subsection{WISE Data}

The Wide-field Infrared Survey Explorer (WISE; Wright et al. 2010) conducted a survey of the entire sky in the 3.4, 4.6, 12 and $22 \mu \mathrm{m}$ mid-infrared bandpasses (hereafter $W 1, W 2, W 3$ and $W 4$ ). The WISE AllSky Source Catalog (see explanatory supplement document by Cutri et al. http://wise2.ipac.caltech.edu/ docs/release/allsky/expsup/ includes the photometry of VHS 1256-1257 in the four bands, but does not detect VHS 1256-1257b. The AllWISE Source Catalog contains the deblended photometry of both the primary in the four bands and the secondary in the $W 1$ and $W 2$,

www.ing.iac.es/Astronomy/instruments/liris/liris_ql.
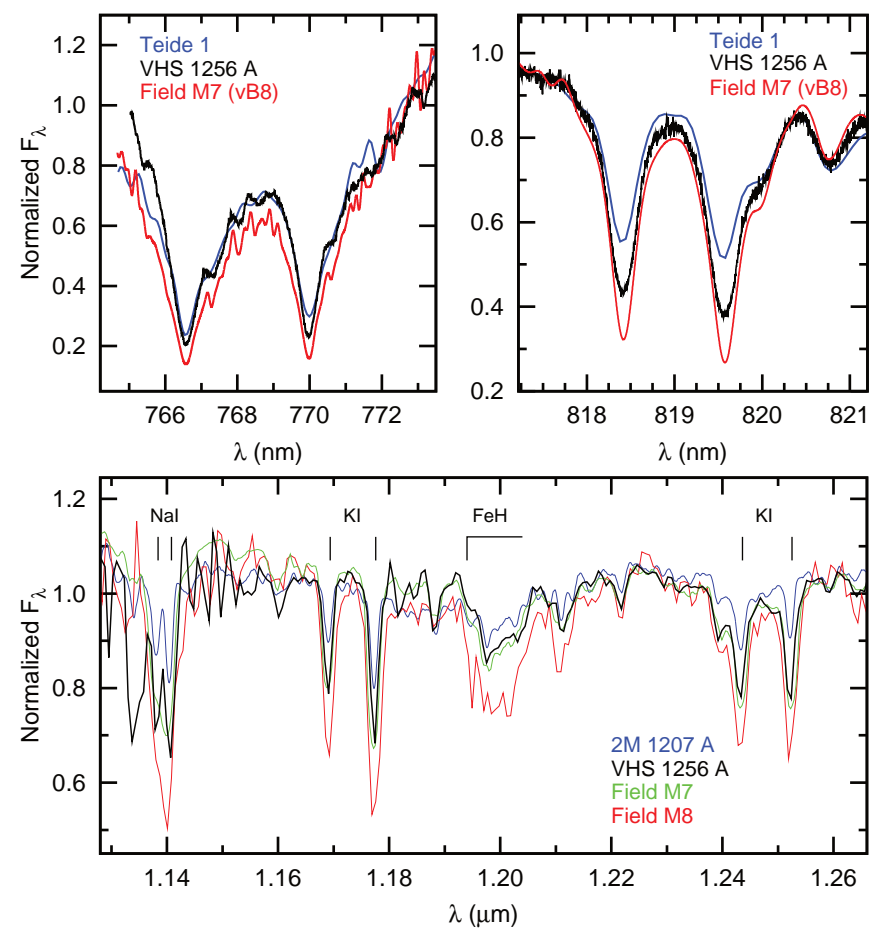

FIG. 5.- Comparison of the NaI and KI lines of the primary VHS 1256-1257 in the optical (top panels) and near-IR (bottom panel) with standard field M7-M8 counterparts and with young low-gravity M8-type dwarfs Teide 1 and 2M 1207 A. All spectra per panel were conveniently normalized at a certain wavelength, and lines were convolved to the same resolution for a proper comparison. These absorption features are slightly less intense than in field objects of similar spectral type.

but the later is contaminated by a diffraction spike of the bright star as indicated in the confusion flag. In order to obtain the correct values, we performed aperture photometry of VHS 1256-1257 b using DAOPHOT routine in the $W 1$ and $W 2$-band images after PSF subtraction of the primary using the scaled PSF of nearby stars in the field as references. A small aperture of 5 pixels was used and instrumental magnitudes was transformed into 
apparent magnitudes using the photometry of VHS 12561257. The AllWISE Source Catalog photometry of the primary and the derived photometry of the secondary are given in Table 2 .

\section{PHYSICAL PROPERTIES OF THE SYSTEM}

\subsection{Spectral Types and Spectral Characteristics}

In Figure 3 we plot the merged low-resolution optical $(0.5-1.0 \mu \mathrm{m})$ and near-IR $(1.0-2.4 \mu \mathrm{m})$ spectra of each of the two components of VHS 1256-1257. The spectral energy distribution of the primary (top panel) corresponds to that of mid-/late-M dwarfs, with the highest flux in the $J$ band, broad water vapor absorption bands cutting out the continuum between $J H$ and $H K$ regions and numerous hydride, oxide ( $\mathrm{FeH}, \mathrm{TiO}, \mathrm{VO}, \mathrm{CO})$, and alkali line (Na I, K I) absorption features. Also, a $\mathrm{H} \alpha$ emission line at $656 \mathrm{~nm}$ is detected. The overall appearance of the companion spectra (bottom panel) appears to resemble an L-type object, but it is significantly redder than field L dwarfs. Unlike a typical L dwarf, its flux peaks at the $H$ band, which has a triangular continuum shape, usually interpreted as a hallmark of low surface gravity and youth. In the following, we discuss in detail the spectral characteristics, determine the spectral types of the binary components, and analyze the gravity-sensitive features in the spectra.

\subsubsection{The Primary}

To determine the spectral types, we used our lowresolution optical and near-IR spectra. We classified the objects in a qualitative manner through visual comparison of the spectra with a set of field dwarf spectral templates, in the optical and near-IR separately. Subsequently, we used the spectral indices established by Allers \& Liu (2013) to designate spectral types in a quantitative way, and estimate the gravity class. The final spectral types were then assigned by averaging the information from both approaches with their corresponding uncertainties.

In Figure 4 we show the optical (left plot) and near-IR (right plot) spectra of the primary overplotted with the templates. In the optical, we compared our GTC/OSIRIS spectra normalized at $900 \mathrm{~nm}$ with a set of M dwarf spectral templates: Gl 406 (M6, Reid et al. 2008), vB 8 (M7, Kirkpatrick et al. 1997) and vB 10 (M8, Henry \& Kirkpatrick 1990). In this wavelength range we find the best match with an M7-type dwarf, while a noticeably poorer fit to templates with one subtype difference. Thus, in the optical we assign a spectral type of $\mathrm{M} 7 \pm 0.5$. In an analogous manner, we compared the near-IR NTT/SofI spectra normalized at $1.6 \mu \mathrm{m}$ with Mdwarf spectral templates available in the IRTF Spectral Library ${ }^{8}$ maintained by Michael Cushing (Rayner et al. 2009). On the right-hand side panel of Figure 4 we include a comparison of the primary spectra with vB 8 (M7, Cushing et al. 2005: Rayner et al. 2009), LP 412-31 (M8, Rayner et al. 2009) all smoothed to match resolution of $R \sim 600$ and the M9 standard LHS 2065 (Kirkpatrick et al. 1991) which we observed using SofI with the same configuration as for VHS 1256-1257. We found the
TABLE 3

Pseudo-equivalent WidThs (PEW) OF LiNES AND DOUblets MEASURED IN THE VLT/UVES, NTT/SOFI AND NOT/ALFOSC SPECTRA OF THE PRIMARY VHS 1256-1257.

\begin{tabular}{lccc}
\hline \hline Line & Date (UT) & $\lambda(\mathrm{nm})$ & $\mathrm{pEW}(\AA)$ \\
\hline $\mathrm{H}_{\eta}$ & 2014 Jul 01 & 383.5 & $-6.06 \pm 0.91$ \\
$\mathrm{H}_{\zeta}$ & 2014 Jul 01 & 388.9 & $-7.94 \pm 1.23$ \\
$\mathrm{Ca}$ II K & 2014 Jul 01 & 393.4 & $-35.63 \pm 4.42$ \\
$\mathrm{Ca}$ II H+H $\epsilon$ & 2014 Jul 01 & $396.8+397.0$ & $-28.44 \pm 3.79$ \\
$\mathrm{H}_{\delta}$ & 2014 Jul 01 & 410.2 & $-9.49 \pm 1.78$ \\
$\mathrm{H}_{\gamma}$ & 2014 Jul 01 & 434.1 & $-8.43 \pm 1.87$ \\
$\mathrm{H}_{\beta}$ & 2014 Jul 01 & 486.1 & $-7.49 \pm 0.31$ \\
$\mathrm{H}_{\alpha}$ & 2014 Apr 22 & 656.3 & $-3.8 \pm 0.5$ \\
$\mathrm{H}_{\alpha}$ & 2014 May 04 & 656.3 & $-4.0 \pm 0.5$ \\
$\mathrm{H}_{\alpha}$ & 2014 Jul 01 & 656.3 & $-4.04 \pm 0.05$ \\
$\mathrm{H}_{\alpha}$ & 2014 Jul 09 & 656.3 & $-3.74 \pm 0.19$ \\
$\mathrm{H}_{\alpha}$ & 2014 Jul 10 & 656.3 & $-2.80 \pm 0.09$ \\
$\mathrm{Li} \mathrm{I}$ & 2014 Jul 01 & 670.8 & $<0.03$ \\
$\mathrm{~K}_{\text {I doublet }}$ & 2014 Jul 01 & $766.5 / 769.9$ & $9.35 \pm 0.17 / 5.94 \pm 0.16$ \\
$\mathrm{Na}$ I doublet & 2014 Jul 01 & $818.3 / 819.8$ & $1.80 \pm 0.06 / 2.88 \pm 0.06$ \\
$\mathrm{Na}$ I & 2014 Mar 12 & 1139 & $8.2 \pm 0.8$ \\
$\mathrm{~K}$ I doublet & 2014 Mar 12 & $1169 / 1177$ & $3.0 \pm 0.6 / 4.2 \pm 0.7$ \\
$\mathrm{~K}$ I doublet & 2014 Mar 12 & $1244 / 1253$ & $4.0 \pm 0.7 / 3.4 \pm 0.6$ \\
\hline
\end{tabular}

best consistency with the M8 standard. Both M7- and M9-type standards provided significantly worse matches, especially in the $J$ band. We adopt a near-IR spectral type of M $8 \pm 0.5$, one subtype later than the one designated from the optical.

As mentioned above, in parallel to visual comparison of the overall spectral morphology with the standards, we calculated the spectral indices defined by Allers \& Liu (2013). We used the $\mathrm{H}_{2} \mathrm{O}, \mathrm{H}_{2} \mathrm{OD}, \mathrm{H}_{2} \mathrm{O}-1$, and $\mathrm{H}_{2} \mathrm{O}-2$ indexes, optimized to avoid spectral features dependent on the surface gravity of the object and to have a wellestablished correlation with optical spectral types. The $\mathrm{H}_{2} \mathrm{O}$ index, which measures the slope of the steam absorption at the blue end of the $H$ band $(\sim 1.50-1.57 \mu \mathrm{m})$, yields a spectral type of M7.6 \pm 0.4 . From $\mathrm{H}_{2} \mathrm{O}-1$ and $\mathrm{H}_{2} \mathrm{O}-2$ indexes we obtained spectral types of M6.9 \pm 1.1 and M7.9 \pm 0.5 , respectively. Both from the comparison with templates and from the calculated indices, we found the typing to be consistent within the uncertainties. As the final spectral type we adopt an M7.5 \pm 0.5 , obtained as the mean of all the types inferred from visual comparison and from spectral indexes, weighted by their corresponding uncertainties.

To find out if the primary has characteristics of low surface gravity, we inspect the gravity-sensitive spectral features like the Na I and KI lines. In Fig. 5 we present a comparison of K I and Na I doublets in the optical at around 770 and $819 \mathrm{~nm}$, respectively (top panels of the figure), and in the near-IR in a region of the $J$ band (bottom panel). In the optical, we plot in black the UVES spectrum of the primary VHS 1256-1257, in red the standard M7 dwarf vB 8 (using UVES spectra retrieved from the ESO Archive) and in blue the M8 member of the Pleiades, Teide 1 (Rebolo et al. 1996). In the near-IR, our NTT/SofI spectrum of VHS 1256-1257 (black line) is overplotted with the field dwarfs vB 8 (M7), LEHPM 2-436 (M8) observed by us with the same instrumental configuration, and with an M8 at 5-10 Myr, 2M $1207 \mathrm{~A}$ from Bonnefoy et al. (2014). The spectra were smoothed to match the resolution of VHS 1256-1256 data and, in the optical, convolved with a Gaussian function to adjust 

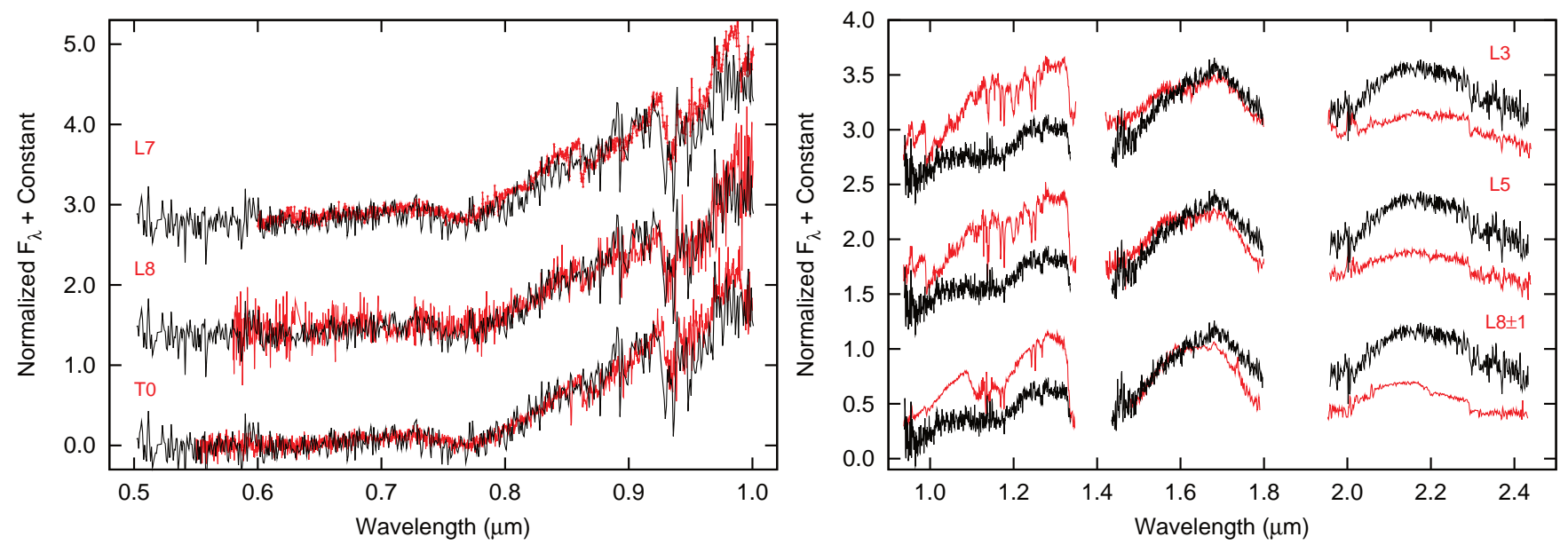

FIG. 6.- Low-resolution GTC/OSIRIS optical (left) and NTT/SofI near-infrared (right) spectra of the companion VHS 1256-1257 b plotted with black line, compared with high-gravity field dwarf templates. The sources and references of used templates are described in Section 4.1.2. Spectra were normalized at $0.9 \mu \mathrm{m}$ at optical and at $1.6 \mu \mathrm{m}$ at near-IR and offset by a constant for display.

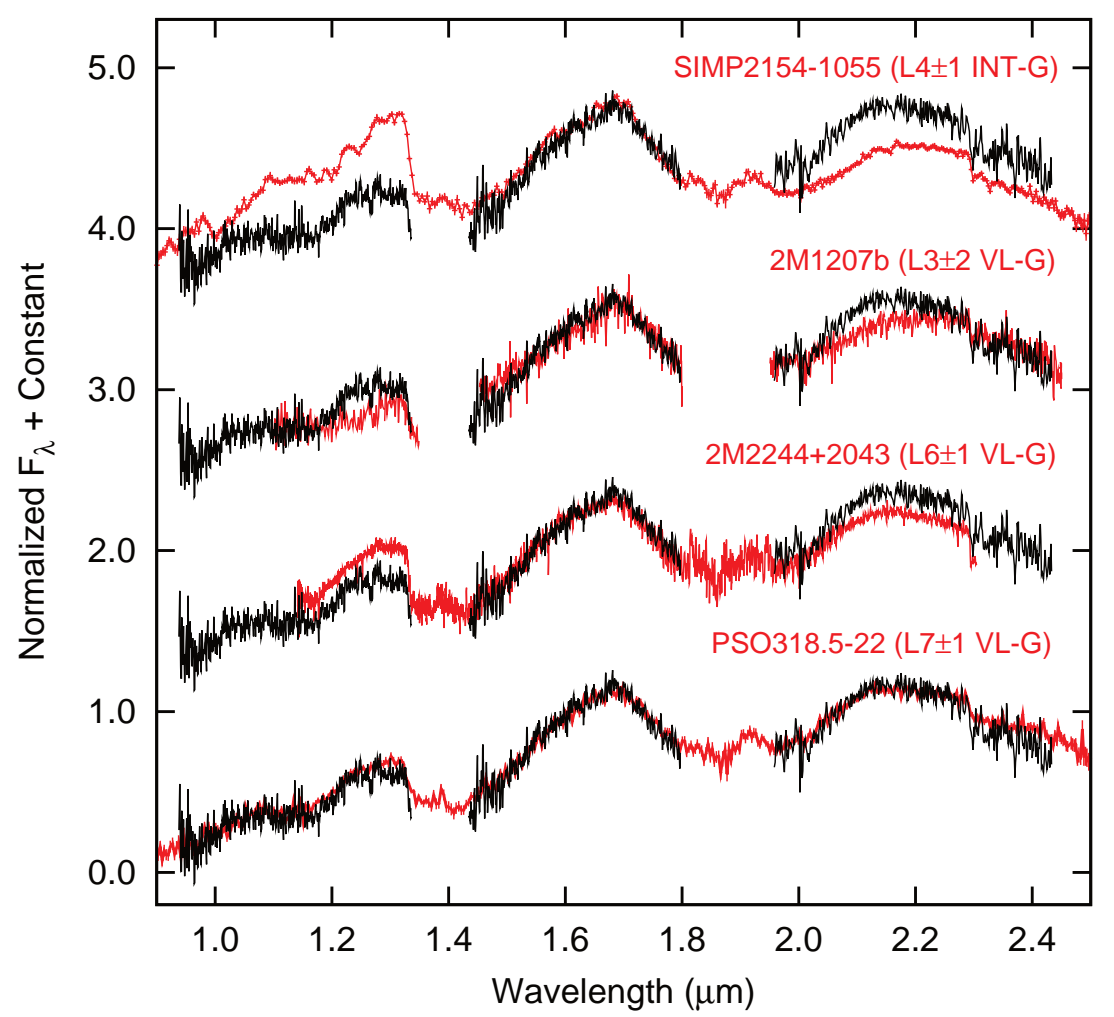

FIG. 7.- SofI/NTT low-resolution near-infrared spectra $(0.9-2.4 \mu \mathrm{m}, R \sim 600)$ of the companion VHS 1256-1257 b (plotted in black) compared with an L4 \pm 1 type dwarf SIMP2154-1055 classified as intermediate surface gravity object (INT-G) and with a set of young, very low-gravity (VL-G) L dwarfs (red lines). Spectra were normalized at $1.6 \mu \mathrm{m}$ and shifted by a constant for display. The spectrum of VHS 1256-1257 b is best matched with spectrum of the free-floating planet PSO J318.5-22 classified as L7 \pm 1 type.

the FWHMs of the lines.

We find these absorption features of the primary to be slightly less intense than in the field counterparts, for example, the $\mathrm{Na}$ I lines at 818.3 and $819.8 \mathrm{~nm}$ have pEW of $1.80 \pm 0.06$ and $2.88 \pm 0.06 \AA$, respectively. In comparison, from the $\mathrm{vB} 8$ spectra we measure $\mathrm{pEW}$ of $1.98 \pm 0.07$ and $3.40 \pm 0.06 \AA$. The same lines in the 120 Myr old M8-type counterpart Teide 1 are in turn less intense with pEW of $1.66 \pm 0.09$ and $2.66 \pm 0.10 \AA$. Because the Na I subordinate lines are well known to be highly sensitive to atmospheric pressure at cool temperatures, this indicates that the primary likely has an intermediate surface gravity that lies between that of Pleiades and field M8-type dwarfs. We note, however, that all these comparisons are based on the assigned spectral types and assume that both targets and reference sources have the same metallicity. Furthermore, the intensity of the alkali lines also depend on the effective temperature (or spectral type). Given a half-subtype uncertainty in our 
typing of VHS 1256-1257 A, we found that the surface gravity of this object may be consistent with field objects considering an earlier type.

In Table 3 we provide the pEW of $\mathrm{Na}$ I, K I, and other lines measured in the optical and near-IR spectra of the primary. In order to quantify the gravity class we have calculated four spectral indices: $\mathrm{FeH}_{z}, \mathrm{VO}_{z}, \mathrm{KI}_{J}$, and $H$-continuum, identified to be related to gravitydependent features and optimized to be employed with low-resolution near-IR spectra Allers \& Liu 2013 and references therein). Based on these indicators we considered field gravity (FLD-G), intermediate gravity (INT$\mathrm{G}$ ), or very low gravity (VL-G) of an object following the classification scheme of Allers \& Liu (2013). For $\mathrm{FeH}_{z}$, which measures the depth of the FeH feature at $0.99 \mu \mathrm{m}$, we obtained a value of $1.13 \pm 0.08$. For the $\mathrm{VO}_{z}$ index we assign a score of " $\mathrm{n}$ " since it is not gravity sensitive in this range of spectral types. For the $\mathrm{K}_{J}$ index, which measures the depth of potassium absorption at 1.244 and $1.253 \mu \mathrm{m}$, we got a value of $1.08 \pm 0.07$ and for the $H$ cont index, which measures the slope of the $H$ band we got $0.98 \pm 0.24$. For an M7.5 spectral type these index values correspond to a surface gravity intermediate between that of counterparts classified as very low gravity and of the old, field population.

\subsubsection{The Secondary}

Our GTC/OSIRIS optical spectrum of the companion (left plot of Fig. 6) is of modest signal-to-noise ratio, and apart from the general spectral energy distribution only few spectral features are noticeable, like the strong KI resonance doublet and water vapor at $0.92 \mu \mathrm{m}$. The lack of oxide features ( $\mathrm{TiO}, \mathrm{VO})$ at the resolution of the visible spectrum suggest a type later than mid-L. On the left panel of Fig. 6 we show the spectra of the companion (black line) normalized at $0.9 \mu \mathrm{m}$ and overplotted with objects of spectral types L7 (2MASS J21522609+0937575, Reid et al. 2008), L8 (2MASS J03400942-6724051, Reid et al. 2008) and T0 (2MASS J04234858-0414035, Cruz et al. 2003). The template spectra were retrieved from the Ultracool RIZzo Spectral Library. Comparison with templates of field dwarfs yields a spectral type of $\mathrm{L} 8 \pm 2$.

From the comparison of the NTT/SofI near-IR spectrum of the companion VHS 1256-1257 b to L-type objects with well-determined spectral types, we find that the overall 1.0-2.4 $\mu \mathrm{m}$ spectral energy distribution does not match any early to late high-gravity field $\mathrm{L}$ dwarf. In the right-hand side plot of Fig. 6 we show a comparison of the companion near-IR spectra normalized at $1.6 \mu \mathrm{m}$ (black line) with an L3-type object DENIS-P J1058.71548 (Burgasser et al. 2010), L5 SDSS J053951.99005902.0 (Cushing et al. 2005) and L8 \pm 1 WISE 10495319 A (Luhman 2013). The spectra of these L dwarf templates were obtained by us using SofI instrument with the same configuration as for VHS 1256-1257 and were reduced in the same way. In the $J$ band, VHS 1256 $1257 \mathrm{~b}$ is relatively less luminous than normal field $\mathrm{L}$ dwarfs and contrarily is significantly brighter in the $K$ band. Its $H$-band continuum unlike the field $\mathrm{L}$ dwarfs has a distinctive triangular shape, interpreted as a signature of low surface gravity and youth. To measure the near-IR spectral type, we tried to find best matching field dwarf standards from the IRTF library, at the
$J(1.07-1.40 \mu \mathrm{m})$ and $K(1.90-2.20 \mu \mathrm{m})$ windows separately, following the approach of Allers \& Liu (2013). In this wavelength regions the shape of spectra continuum is expected to have a lower dependence on gravity. We estimate, in the $J$ and $K$ bands, respectively, a spectral type of $\mathrm{L} 8 \pm 1$ and $\mathrm{L} 5 \pm 2$. In the $K$ band we assign an uncertainty of two subtypes because several standards provide similarly good fits.

Along with visual comparison, we calculated the $\mathrm{H}_{2} \mathrm{OD}$ index, well defined up to the range of late-L dwarfs. The other indexes are not valid for spectral types later than L5. The $\mathrm{H}_{2} \mathrm{OD}$ index value give a spectral type of L5.8 \pm 0.8 . In analogous manner as for the primary, from a mean of the types inferred through visual comparison and from the $\mathrm{H}_{2} \mathrm{OD}$ spectral index, weighted by the corresponding errors, we obtained an L7.0 type, which we adopt here as the spectral type of the companion, with an uncertainty of 1.5 subtype. Among the gravitysensitive spectral indices, only the $H$-continuum index is well defined up to L7 type objects. For VHS 1256-1257 b $H$-cont indicates a very low gravity (VL-G). However, as noted by Allers \& Liu (2013), the shape of the $H$-band continuum is not the most reliable indicator, and can provide rather only a hint of low gravity. It should be used in combination with other gravity-sensitive indices, but for the spectral type in question none of them have yet been defined. The $\mathrm{K}$ I lines at $1.17 \mu \mathrm{m}(\mathrm{pEW} \leq 5 \AA)$ and at $1.25 \mu \mathrm{m}(\mathrm{pEW} \leq 3 \AA)$ appear weaker in the spectrum of the secondary than in the field mid- and late-L dwarfs (see Fig. 6). For example, in the spectrum of the L8 dwarf WISE 1049-5319 A obtained with the same instrument and setup we measured a pEW of the $\mathrm{K}$ I lines of $5.7 \pm 0.8$ and $6.8 \pm 0.7 \AA$ at 1.169 and $1.177 \mu \mathrm{m}$ and $3.4 \pm 0.4$ and $6.1 \pm 0.4 \AA$ at 1.244 and $1.253 \mu \mathrm{m}$, respectively. This also indicates that the secondary has a lower gravity compared to field counterparts (?).

In addition to the comparison with field L-type templates, we have also compared the near-IR spectra of VHS 1256-1257 b with a set of known young low-gravity mid- and late-L dwarfs. In Figure 7 we overplot the companion spectra with SIMP J2154-1055, classified as an L4 $\pm 1 \beta$ with intermediate surface gravity Gagné et al. 2014), a $\sim 5 M_{\text {Jup }}$ planetary mass companion 2MASS 1207-3932 b at 8 Myr (Chauvin et al. 2005, Patience et al. 2010), 2MASS J22443167+2043433 classified as very low-gravity L6 \pm 1 type (McLean et al. 2003) and with the free-floating planetary mass object PSO J318.5-22 (Liu et al. 2013). In general, the spectrum of the companion fits much better to the spectra of young, very low-gravity $\mathrm{L}$ dwarfs than to the spectra of field objects. We find the best match with PSO J318.5-22 which is classified as an $\mathrm{L} 7 \pm 1$, thus providing a further support of our spectral type classification. PSO J318.5-22 is a $6.5 M_{\text {Jup }}$ object, possible member of the $12 \mathrm{Myr}$ moving group $\beta$ Pictoris. Because of this strong resemblance, we state that the companion can be assigned to the very low-gravity (VL-G) class.

\subsection{Distance Estimates}

\subsubsection{Spectrophotometric Distance}

Having classified the spectral type of the primary, we used the $J H K_{s}$ photometry to estimate the distance of the system. We used the 2MASS catalog photometry, 


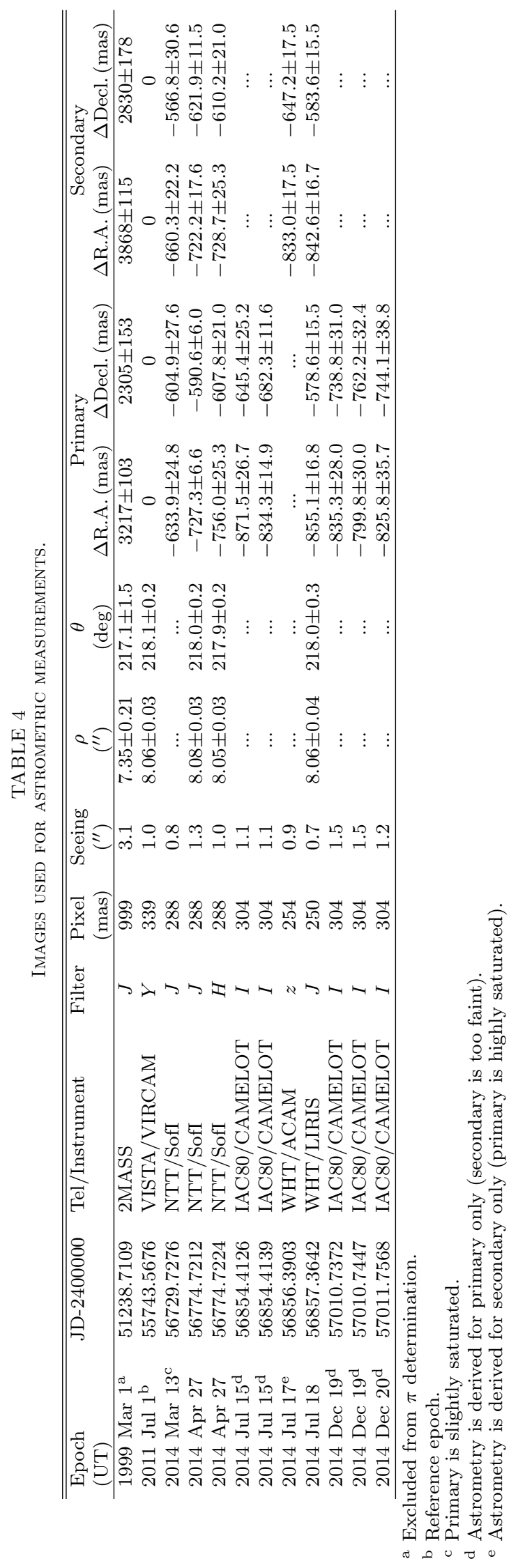


since at these magnitudes the VHS measurements start to get beyond the linear regime of the detector and could be uncertain. We employed the mean absolute magnitudes as a function of spectral type determined by Dupuy \& Liu (2012) from a set including 8 M7.0, 9 M7.5, and 11 M8.0 type dwarfs with precise parallactic distance determination. Considering an M7.5 \pm 0.5 type of the primary, and assuming that it is a single object, we inferred spectroscopic distances of $12.0_{-3.6}^{+5.8}, 12.4_{-3.5}^{+4.7}$ and $12.2_{-3.3}^{+4.3} \mathrm{pc}$, using $J, H$, and $K_{s}$ photometry, respectively. Taking the average of three bands, we estimate a distance of $12.2_{-3.5}^{+5.0} \mathrm{pc}$. The quoted errors account for uncertainties of the photometry, spectral type, and the intrinsic dispersion in absolute magnitudes for field dwarfs at a given spectral type.

In this case however, the estimation that employs the M dwarfs found in the field should be considered with caution, since the field objects have ages typically of more than 1 Gyr, and for VHS 1256-1257 we expect an age below $300 \mathrm{Myr}$ (see Section 4.5). From the studies of young star clusters, it is known that the M dwarfs younger than 400-625 Myr can be overluminous with respect to their field counterparts (Bihain et al. 2006 Jameson et al. 2008, Zapatero Osorio et al. 2014c). For that reason we obtained an alternative estimation using near-IR photometry of M7-M8 dwarf members of the Pleiades cluster, compiled by Bihain et al. (2010). The Pleiades has an age of $120 \pm 10 \mathrm{Myr}$ (Basri et al. 1996 Martín et al. 1998: Stauffer et al. 1998) and is located at a distance of $133.5 \mathrm{pc}$ (Soderblom et al. 2005; Melis et al. 2014). Averaging the estimates obtained in $J, H$, and $K_{s}$ yields a distance of $14.9_{-3.2}^{+3.3}$ pc for VHS 1256-1257 if this binary had the age of the Pleiades.

\subsubsection{Trigonometric Parallax}

To derive the trigonometric parallax and proper motion of the pair we used an area of $5^{\prime} \times 5^{\prime}$ around the target and the images listed in Table 4 except for the 2MASS. We took advantage of the availability of these images for a first determination of a trigonometric distance to the system. In Table 4 we provide the observing epoch (including the Julian Date), telescope, and imaging instrument, filter, the average pixel projection onto the sky, and the mean seeing of the images. All data were properly reduced following standard steps for the optical and near-IR wavelengths as explained in Section 3. We selected the VISTA/VIRCAM $Y$-band observations of 2011 July as the fundamental frame to which all other images are compared. Using the DAOFIND command within IRAF we identified all sources with photon peaks with detection above $6 \sigma$, where $\sigma$ stands for the noise of the background, and FWHM resembles that of unresolved objects (i.e., extended sources were mostly avoided). In addition, we ensured that the detected sources lied within the linear regime of the detectors response (with the only exception of the primary component of the binary). The number of sources identified per image in common with the reference frame ranged from 25 to $\sim 40$. The centroids of detected objects were computed by estimating the $x$ and $y$ pixel positions of the best fitting one-dimensional Gaussian functions in each axis; typical associated errors are about $3 \%-5 \%$ of a pixel or better.

Pixel coordinates were transformed between different

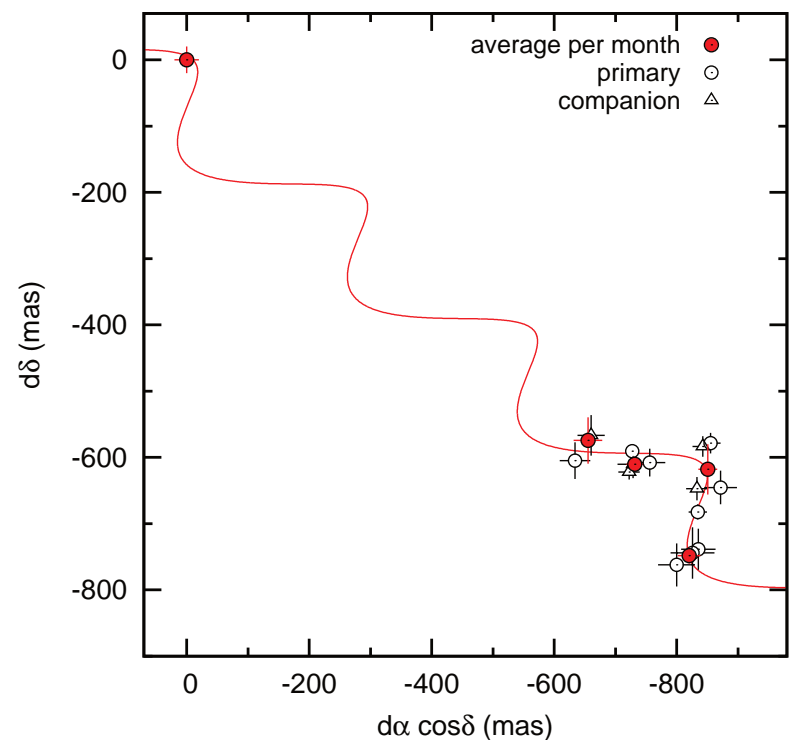

FIG. 8.- Relative apparent trajectory of the components of VHS 1256-1257 in right ascension $(\alpha)$ and declination $(\delta)$ coordinates for the time interval 2011 July (reference epoch) through 2014 December. Each component is plotted separately as indicated in the figure legend, and the averaged values per observing month are shown with the red solid circles. The solid line indicates the best model fit. North is up and east is to the left.

epochs using the GEOMAP routine within IRAF, which applied a polynomial of the third and fourth order in $x$ and $y$ and computed linear terms and distortions terms separately. The linear term included an $x$ and $y$ shift and an $x$ and $y$ scale factor, a rotation, and a skew. The distortion surface term consisted of a polynomial fit to the residuals of the linear term. The $(x, y)$ astrometric transformation between observing epochs and the reference epoch was an iterative step, which included the rejection of objects deviating by more than 1.5-2 $\sigma$, where $\sigma$ corresponds to the dispersion of the transformation. Typical coordinates transformation dispersions ranged from \pm 0.05 to \pm 0.09 pixels; this is typically $\pm 15-25$ mas. The centroids of the two components were calculated with an uncertainty similar to that of the astrometric reference sources. Errors associated with the astrometry are dominated by the errors in the coordinate transformations for each axis (R.A., decl.), which include the dispersion of all reference sources. We therefore assigned the dispersion of the coordinates transformation solutions to the errors associated with the differential astrometry of our targets listed in Table 4 . We did not apply any correction for differential chromatic refraction since all of our data were taken at red wavelengths, where the corrections are smaller than the quoted astrometric uncertainty. The relative $(d x, d y)$ astrometry (conveniently derotated) was converted into $\mathrm{d} \alpha \cos \delta$ and $\mathrm{d} \delta$ using the corresponding plate scales. The apparent trajectory of each component, which depends on proper motion $\left(\mu_{\alpha}, \mu_{\delta}\right)$ and parallax $(\pi)$, is shown in Figure 8 and can be modeled with the following equations:

$$
\begin{aligned}
& \mathrm{d} \alpha=\mu_{\alpha}\left(t-t_{o}\right)+\pi\left(f_{t}^{\alpha}-f_{o}^{\alpha}\right) \\
& \mathrm{d} \delta=\mu_{\delta}\left(t-t_{o}\right)+\pi\left(f_{t}^{\delta}-f_{o}^{\delta}\right),
\end{aligned}
$$

where $t$ stands for time, the subscript $o$ indicates the reference epoch, and $f^{\alpha}$ and $f^{\delta}$ refer to the parallax fac- 
tors in right ascension $(\alpha)$ and declination $(\delta)$, respectively. In our study, all the astrometric quantities are given in mas and the times $t$ and $t_{o}$ are measured in Julian Days. The parallax factors were computed by following the equations given in Green (1985) and obtaining the Earth barycenter from the DE405 Ephemeris 9 We applied the least-squares fitting method to the set of Equations 1] and 2 to derive the parallax and the proper motion of the system. The two components were fit simultaneously. The best-fit solution yielded $\mu_{\alpha} \cos \delta=-277 \pm 5$ mas yr $^{-1}, \mu_{\delta}=-203 \pm 12.5$ mas yr $^{-1}$

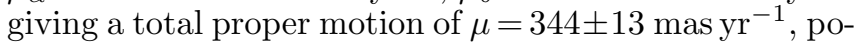
sition angle of the proper motion vector of $233.7^{\circ} \pm 2.5^{\circ}$, and a relative parallax $\pi=77.79 \pm 6.4$ mas. It is depicted with a solid curve in Figure 8. The amplitude and position angle of the proper motion we determined is in agreement with the measurement given by Deacon \& Hambly (2007). From the fitting performed for each pair member individually we obtained proper motion of $\mu_{\alpha} \cos \delta=-281.5 \pm 5.3$ mas yr$^{-1}, \mu_{\delta}=-205.5 \pm$ 15.2 mas yr $^{-1}$ for the primary and $\mu_{\alpha} \cos \delta=-275.4 \pm$ 5.3 mas yr$^{-1}, \mu_{\delta}=-198.4 \pm 15.2$ mas yr$^{-1}$ for the companion. The above values are listed in Table 2. We have also tested the WISE, 2MASS, and available earlier epoch data (DENIS, USNO-B1.0, DSS) in the proper motion and parallax determination, but despite the longer baseline they introduced larger errors to the fitted values. Hence, for the determination of the parallax and proper motion we excluded these data, given that their associated uncertainties are at least one order of magnitude higher than those of the recent data. We remark that given the time coverage of our data, which span 3.0447 $\mathrm{yr}$, the proper motion is more precisely determined than the parallax. Additional images, in particular taken on months that were not covered yet, are required for decreasing the uncertainty in the parallax measurement.

We did not correct our proper motion for the motions of the stars used as a reference because we assumed that these (small) motions are randomly orientated with a negligible net effect. However, we did apply the correction for converting the relative parallax into the absolute parallax. This takes into account that the reference stars are located at finite distances, which diminish part of the true parallax of our targets. We followed a procedure similar to the one described in Faherty et al. (2012) and Zapatero Osorio et al. (2014a). Using the 2MASS colors (Skrutskie et al. 2006) of the reference objects, we obtained their photometric distances by assuming that all of them are main sequence stars. We adopted the color-bolometric correction-spectral type relations given in Johnson (1966) for BAFGK stars and in Kirkpatrick et al. (1993) for late-K and $M$ stars. The defined relations are valid for colors in the interval $J-K_{s}=-0.2$ to $1.53 \mathrm{mag}$. We adopted the mode of the distribution of reference objects distances as the correction to be added to the relative parallax that comes directly from our fit to obtain the absolute parallax. The absolute parallax is $\pi=78.79 \pm 6.4$ mas, which translates into a distance of $12.7 \pm 1.0 \mathrm{pc}$, consistent with the value from the spectrophotometric estimates using field counterparts. This suggests that the primary component is not an equalmass binary.

\footnotetext{
9 http://ssd.jpl.nasa.gov
}

\subsection{Angular Separations and Orbital Motion}

On the available images where both components were detected and the primary did not saturate we measured the projected angular separation $(\rho)$ and position angle $(\theta)$ of the companion using the centroid positions of the two sources in each of the images transformed into the VHS $\alpha, \delta$ coordinates as explained above. The determined values are given in Table 4. The angular separations and position angles of VHS 1256-1257 in the most recent epoch images over the last three years are consistent within $\delta \rho<30$ mas and $\delta \theta<0.04 \mathrm{deg}$, respectively. Both components share the same proper motion, and given the low probability to find a very red young $\mathrm{L}$ dwarf (only a few tens found in the whole sky area) at this very short angular separation of $8 . .06$, we conclude that both objects are gravitationally related. These results also indicate that we do not detect an orbital motion higher than 10 mas yr $^{-1}$ (at the $1 \sigma$ level).

We expect an orbital period of about 3900 yr from the estimated masses of the primary and companion (Section 4.7) and a physical projected separation of $\sim 102 \mathrm{AU}$. Assuming a circular orbit, this implies that the displacement caused by orbital motion would be of 4-13 mas yr ${ }^{-1}$ depending on the orientation of the orbit, which is consistent with our results. The angular separation of the pair in the 2MASS images is lower, although consistent within $3 \sigma$. These differences $\left(0{ }^{\prime \prime} 6 \pm 0\right.$ '! 2$)$ can not be explained by the orbital motion of the companion, since we expect no more than $\sim 13$ mas yr $^{-1}$ for a face-on circular orbit at the physical separation found, and may be attributed to systematic errors in the determination of the centroids in the 2MASS data probably due to small separation of the components and low resolution of the images.

\subsection{Kinematics}

To measure the heliocentric radial velocity of VHS 1256-1257 we employed the high spectral resolution UVES data (mean Modified Julian Date, $\mathrm{MJD}=56838.3530)$ and the cross-correlation method against the M6V star GJ 406, which has a known, constant radial velocity of $v_{h}=19.5 \pm 0.1 \mathrm{~km} \mathrm{~s}^{-1}$ (Nidever et al. 2002) and a small projected rotational velocity of $v \sin i \leq 3 \mathrm{~km} \mathrm{~s}^{-1}$ (Reiners \& Basri 2010). GJ 406 was also observed with the VLT/UVES instrument (spectral resolution of $\sim 45,000$ ) on 2009 March 14 $(\mathrm{MJD}=54904.0839)$. We downloaded the reduced UVES spectrum of GJ 406 from the ESO data archive (program 082.D-0953). Although GJ 406 was observed with a nonstandard instrumental setup in terms of wavelength coverage (see Fuhrmeister et al. 2011), the overlap between the M6V-type star and VHS 1256-1257 data is significant and secures a reliable velocity determination. The telluric contribution was removed from the data of GJ 406 in the same manner as we did for VHS 1256-1257 (see Section 3.4).

The telluric-free UVES spectra of VHS 1256-1257 and GJ 406 were cross-correlated using the task FXCOR within IRAF. We fit a Gaussian function to the peak of the cross-correlation distribution. The resulting relative displacement was corrected for the lunar, diurnal, and annual velocities to obtain the heliocentric radial velocity of VHS 1256-1257. All UVES spectra were calibrated in 
wavelength using ThAr arc lines taken some time after the science observations (Section 3.4). The typical stability of UVES along the spectral axis is on the order of a pixel over the course of a night; therefore, deviations of a $\mathrm{km} \mathrm{s}^{-1}$ can be expected. To correct our velocity determination for this effect, we employed the absorption lines from the Earth's atmosphere present in the data, which provide a reliable reference frame within \pm 90 $\mathrm{m} \mathrm{s}^{-1}$ (e.g., Griffin \& Griffin 1973). We used the UVES spectra of VHS 1256-1257 and GJ 406 before subtraction of the sharp telluric lines to determine the instrumental shifts of the wavelength solution for each object. We found them to be $+0.62 \pm 0.20 \mathrm{~km} \mathrm{~s}^{-1}$ (VHS 12561257 ) and $-0.14 \pm 0.20 \mathrm{~km} \mathrm{~s}^{-1}$ (VHS 1256-1257 versus GJ 406). The final corrected heliocentric radial velocity of VHS 1256-1257 is $v_{h}=-1.4 \pm 5.0 \mathrm{~km} \mathrm{~s}^{-1}$, where the error bar accounts for the uncertainties due to the crosscorrelation procedure, the corrections of the wavelength reference frames, and the error associated with the velocity of the M6V standard star.

We checked this heliocentric velocity using the line centroid method, which is independent of any reference star. By measuring the centroids of alkali and other metallic lines in the three UVES spectra of VHS 1256-1257 and considering the correction for the wavelength reference frame and lunar, diurnal, and annual velocities as previously discussed, we found $v_{h}=+4.1 \pm 4.0 \mathrm{~km} \mathrm{~s}^{-1}$. Albeit having a much lower spectral resolution, the NOT/ALFOSC spectra of VHS 1256-1257 also yielded radial velocities that agree with the UVES result at the $1 \sigma$ level (where $\sigma$ stands for the velocity uncertainty): $v_{h}=+0.1 \pm 7.0 \mathrm{~km} \mathrm{~s}^{-1}(\mathrm{MJD}=56769.0443)$ and $+14.1 \pm 10.0 \mathrm{~km} \mathrm{~s}^{-1}(\mathrm{MJD}=56781.9229)$. The ALFOSC velocities were derived using the cross-correlation technique; the M4.5V star GJ $388\left(v_{h}=+12.6215 \mathrm{~km} \mathrm{~s}^{-1}\right.$, Bonfils et al. (2013); $v_{h}=+12.453 \mathrm{~km} \mathrm{~s}^{-1}$, Chubak et al. 2012) acted as the velocity reference source because its spectra were acquired with exactly the same instrumental configuration and observing dates as our target (Section 3.3). GJ 388 was found to be a radial velocity variable star by Bonfils et al. (2013). However, the amplitude of the variations is $0.25 \mathrm{~km} \mathrm{~s}^{-1}$, which is about $\geq 30$ times smaller than the velocity precision we were able to achieve with the ALFOSC data. No obvious velocity change larger than $\sim 10 \mathrm{~km} \mathrm{~s}^{-1}$ is observed in VHS 12561257 over the 69 day interval of spectroscopic observations.

Having the measurements of proper motion, parallax, and radial velocity we can determine the three components of the Galactic space velocity, $U, V$, and $W$. We applied the formulas presented by Johnson \& Soderblom (1987) to derive the $U V W$ velocities listed in Table 2 and displayed in Figure 9. The uncertainties associated to all three Galactic velocities come from the proper motion, parallax, and radial velocity error bars. Figure 9 also illustrates the ellipsoids corresponding to well-known young stellar moving groups of the solar neighborhood (data compiled from Zuckerman \& Song 2004 and Torres et al. 2008).

As seen in Figure 9 , the space velocities of VHS 12561257 fits with the $\beta$ Pic Group and is consistent within error bars with the galactic velocities of Taurus-Auriga, TW Hya, and the Local Association groups. The stars


FIG. 9.- Galactic space velocities of VHS 1256-1257 (red dots) with overplotted ellipsoids of known young star associations and moving groups. Errors incorporate uncertainties in the proper motion, parallactic distance, and radial velocity. Galactocentric $U$ velocity is positive toward the Galactic center.

in $\beta$ Pic, Taurus-Auriga, and TW Hya are younger than $20 \mathrm{Myr}$, and this age is incompatible with the nondetection of lithium in the atmosphere of the primary (see next section). Hence the kinematic and spectroscopic properties of VHS 1256-1257 is only compatible with the membership to the Local Association Group, a coherent kinematic stream of young stars (all below 300 Myr, Eggen 1992) with constituent clusters and associations such as the Pleiades (120 Myr), $\alpha$ Persei (50-80 Myr), and IC 2602 ( $\sim 70 \mathrm{Myr})$. Membership in stellar moving groups is commonly used to constrain or confirm the ages especially of young $(<1 \mathrm{Gyr})$ objects. We discuss in more detail the age of VHS 1256-1257 system in the following section.

\subsection{Activity, Lithium and Age}

The high-resolution optical spectra of the primary (Fig.10,11) reveals emission features recognized as indicators of chromospheric activity in very low-mass stars. We detect the Balmer series emission lines of hydrogen, from $\mathrm{H}_{\alpha}$ up to $\mathrm{H}_{\eta}$, and single ionized calcium Ca II $\mathrm{H}$ and $\mathrm{K}$ emission lines at 396.8 and $393.4 \mathrm{~nm}$. Measurements of the lines pEW are given in Table 3 . The $\mathrm{pEW}$ of $\mathrm{H}_{\alpha}$ does not show a strong variability in timescales of days and months, but show small variations of the order of 1-2 $\AA$ along the observations, as can be seen in Fig.11 and Table 3. These activity indicators of the primary are consistent with dwarfs of similar spectral types in the field and in intermediate age clusters like the Hyades (Reid \& Hawley 1999, West et al. 2011), and are less active than those of younger open clusters like Pleiades (Stauffer et al. 1998).

In the VLT/UVES spectrum of VHS 1256-1257, we did not detect the $\mathrm{Li}$ I resonance doublet at $670.8 \mathrm{~nm}$, imposing an upper limit of $30 \mathrm{~m} \AA$ in the $\mathrm{pEW}$ of this atomic 



FIG. 10. - VLT/UVES spectra of the primary VHS 1256-1257 covering 373-495 and 565-950 nm wavelength range at a resolution of $R \sim 40000\left(25 \mathrm{~mA} \mathrm{pix}^{-1}\right)$. The blue part of the spectrum (left panel) was smoothed for the display by a factor of 33 , degrading the resolution to $R \sim 10000$. The spectrum has been corrected for telluric absorptions using the ESO Molecfit software. Certain features indicating strong magnetic activity in the chromosphere are visible: the Balmer series emission lines from $\mathrm{H}_{\alpha} \mathrm{up}$ to $\mathrm{H}_{\eta}$, singly ionized calcium $\mathrm{H}$ and $\mathrm{K}$ lines at 396.85 and $393.37 \mathrm{~nm}$, respectively.

line (see the right panel of Fig.11). This is much lower than the expected value for a full preservation of this element in the atmosphere of late-M dwarfs of $\mathrm{EW}=0.5-$ $1 \AA$ (Stauffer et al. 1998: Zapatero Osorio et al. 2002). The Li element is rapidly destroyed in the interior of stars, on timescales shorter than $\sim 150 \mathrm{Myr}$, and in massive brown dwarfs on timescales of a few Gyr. Brown dwarfs with masses lower than $0.055-0.060 M_{\odot}$ do not burn this element in their interiors because their central temperature is not high enough to produce this fusion reaction (Ushomirsky et al. 1998 Chabrier et al. 2000b). The non-detection of $\mathrm{Li}$ in VHS 1256-1257 impose a lower limit in the mass of the primary of $0.055^{-}$ $0.060 M_{\odot}$ and also imposes a lower limit to the age of the system, since objects of similar spectral type in the Pleiades cluster (age $\sim 120 \mathrm{Myr}$ ) have fully preserved this element (Stauffer et al. 1998). Theoretical evolutionary models (Chabrier \& Baraffe 1997: Chabrier et al. $2000 \mathrm{~b}$ predict that objects with effective temperature $T_{\text {eff }} \sim 2600 \mathrm{~K}$ (which is the corresponding effective temperature of an M7.5) have preserved their Li content in timescales of less than $150 \mathrm{Myr}$, but have destroyed it for several orders of magnitude at ages larger than $200 \mathrm{Myr}$.

The gravity-dependent spectral features of the primary like $\mathrm{Na}$ and $\mathrm{K}$ alkaline lines are also consistent with intermediate gravities between those of the Pleiades and field dwarfs (Gorlova et al. 2003; Allers \& Liu 2013; Bonnefoy et al. 2014: Gálvez-Ortiz et al. 2014) On the other hand, the secondary shows spectral features, which are signposts of low gravity and youth like the sharp triangular shape of the $H$ band. Some authors attribute these features to objects with ages younger than $150 \mathrm{Myr}$ (Cruz et al. 2009; Faherty et al. 2013a), which is in contradiction with the lower limit to the age of VHS 1256-1257, given by the non-detection of $\mathrm{Li}$ in the primary. Other authors suggest a more conservative range of ages up to 120-500 Myr (Zapatero Osorio et al.|2014a), which are in good agreement with the age constrain for this system.

The proper motion, radial velocity, and parallactic distance of the primary allow us to determine its galactic kinematic. The galactic velocities UVW of the VHS 1256-1257 indicate that the system probably belongs to the Local Association, whose members have estimated ages of 10-300 Myr (Asiain et al. 1999). In conclu-

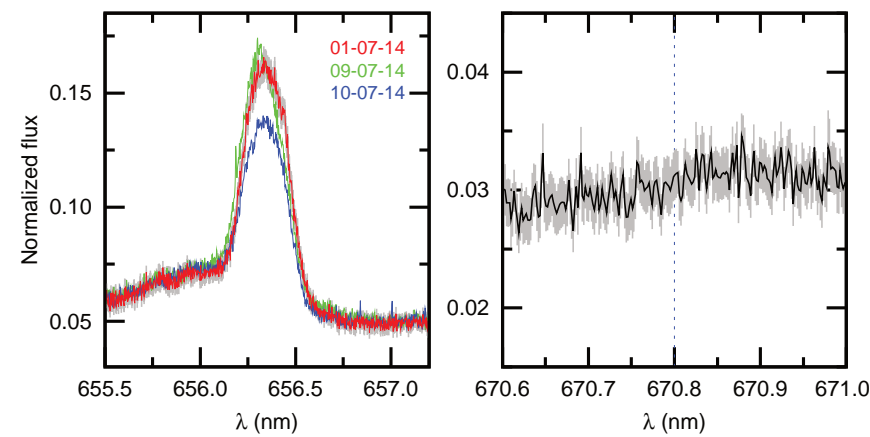

FIG. 11. - Close-up of regions of the VLT/UVES spectrum of the primary VHS 1256-1257, showing, in the left panel the H $\alpha$ emission line at $656.3 \mathrm{~nm}$ and in the right panel the non-detection of lithium absorption at $670.8 \mathrm{~nm}$. Flux uncertainties are plotted in gray.

sion, based on the absence of lithium in the primary and the likely membership to the Local Association, we adopt a range of 150-300 M yr for the age of the VHS 1256-1257 system.

\section{6. $M_{J, K s}$ Versus $J-K_{s}$ Color-Magnitude Diagrams}

Average near-IR $J-K_{s}$ color of L7 dwarfs is $1.75 \pm 0.26$ (Faherty et al. 2013b). With $J-K_{s}=2.47 \pm 0.03 \mathrm{mag}$, VHS 1256-1257 b is among the reddest known L dwarfs, next to 2M1207 b and PSO J318.5-22. These extreme colors are thought to originate from enhanced photospheric dust and broad-band changes in the spectral energy distribution induced by the low gravity of young objects. In Figure 12 we compare the absolute $J$ and $K_{s}$ magnitudes versus $J-K_{s}$ colors of VHS 1256-1257 with field mid-late-M, L and T dwarfs with measured parallaxes compiled by Dupuy \& Liu (2012) and with several known substellar objects and giant planet companions. Based on parallactic distance we derived an absolute magnitude of the companion $M_{J}=16.45 \pm 0.30$, which is about $2.2 \mathrm{mag}$ fainter than the late-L field counterparts. In the $K_{s}$ band it is roughly one magnitude below the LT sequence. The location of VHS 1256-1257 b on the $M_{J}$ and $M_{K s}$ versus $J-K_{s}$ color magnitude diagrams coincides with the young planetary mass companion $2 \mathrm{M} 1207 \mathrm{~b}$ and is almost the same as the $5-11 M_{\mathrm{Jup}}$ planet HR $8799 \mathrm{~b}$. 



Fig. 12.- $M_{J}$ and $M_{K s}$ vs. $J-K_{s}$ color-magnitude diagrams comparing the two components of VHS 1256-1257 with field M, L, and T dwarfs with known parallax measurements from Dupuy \& Liu (2012), known young substellar objects from the compilation of Bowler et al. (2013) and the least-massive Pleiades members (Zapatero Usorio et al. 2014b). All photometry was converted to the MKO filter system.

\subsection{Luminosity, Mass, and Effective Temperature}

To derive the bolometric luminosities of the primary and the companion, we used the near-IR photometry and the parallactic distance. For the primary, which does not deviate strongly from the field sequence, we have obtained the bolometric magnitude applying the corresponding $\mathrm{BC}_{J}$ and $\mathrm{BC}_{K}$ bolometric corrections determined for field objects, from Golimowski et al. (2004) and Dahn et al. (2002). These BCs combined with absolute magnitudes and $M_{\mathrm{bol}}=4.73 \mathrm{mag}$ for the Sun yield the luminosity of $\log \left(L_{\mathrm{bol}} / L_{\odot}\right)=-3.14 \pm 0.10$ dex. The error accounts for the uncertainties in distance, photometry, and bolometric correction. Since the near-IR photometry of the companion differs significantly from the "normal" field L dwarfs, the bolometric corrections determined for field ultracool dwarfs are not valid (Luhman 2012, Faherty et al. 2012, Zapatero Osorio et al. 2014a). We applied bolometric corrections $\left(J H K_{s}, 2 \mathrm{MASS}\right.$ system) derived from the measurements of PSO J318.5-22 (Liu et al. 2013), which shows a strong similarity to VHS 1256-1257b. Taking the mean value from the three bands, we obtained $\log \left(L_{\text {bol }} / L_{\odot}\right)=-5.05 \pm 0.22$ dex.

We used the calculated bolometric luminosities to infer the range of possible masses, effective temperatures, and gravities $(\log g$ ) from the evolutionary models, adopting an age of the system of 150-300 Myr. We have used the Lyon group models BT-Settl (Baraffe et al. 1998, 2003, Chabrier et al. 2000b) with the Caffau et al. (2011) solar abundances and the 'hot start' cloudy atmosphere evolutionary tracks of Saumon \& Marley (2008), with sedimentation parameter $f_{\text {sed }}=2$. Both models give con- sistent values of the derived parameters (masses, $T_{\text {eff }}$, $\log g$ ) within their corresponding uncertainties. For the primary, we obtained a mass of $73_{-15}^{+20} M_{\text {Jup }}$, close to the boundary between stars and brown dwarfs regime which starts at masses below $\sim 75 M_{\text {Jup }}$ for solar metallicities. The effective temperature and $\log g$ found from the models are $2620 \pm 140 \mathrm{~K}$ and $5.05 \pm 0.10 \mathrm{dex}$, respectively. This $T_{\text {eff }}$ is consistent with typical temperatures of field M7.5 \pm 0.5 dwarfs being in the $\sim 2500-2600 \mathrm{~K}$ range (Leggett et al. 2000, Golimowski et al. 2004). The derived gravity is slightly lower but similar to the expected gravity for field dwarfs of similar spectral types $(\log g=5.0-5.5)$. This is consistent with what we have found spectroscopically.

For the companion, we obtained a mass of $11.2_{-1.8}^{+9.7} M_{\text {Jup }}$, indicating that the object is near the mass limit at which the onset of deuterium fusion occurs. Given the uncertainty in the mass determination, it is currently unclear whether the object is above or below it. The effective temperature and $\log g$ of the companion obtained from the evolutionary models are $880_{-110}^{+140}$ $\mathrm{K}$ and $4.24_{-0.10}^{+0.35}$ dex. A typical effective temperature of field L6-L8 dwarf is between 1600 and $1300 \mathrm{~K}$ (Leggett et al. 2000: Golimowski et al. 2004, Vrba et al. 2004). 'The effective temperature of the companion indicated by the models is about $500 \mathrm{~K}$ lower than that and corresponds rather to a field mid-T dwarf. A similar discrepancy between temperatures predicted by evolutionary models and those estimated from spectral classification has been found in other young substellar objects: PSO J318.522 (Liu et al. 2013), 2MASS 0122-2439 B (Bowler et al. 


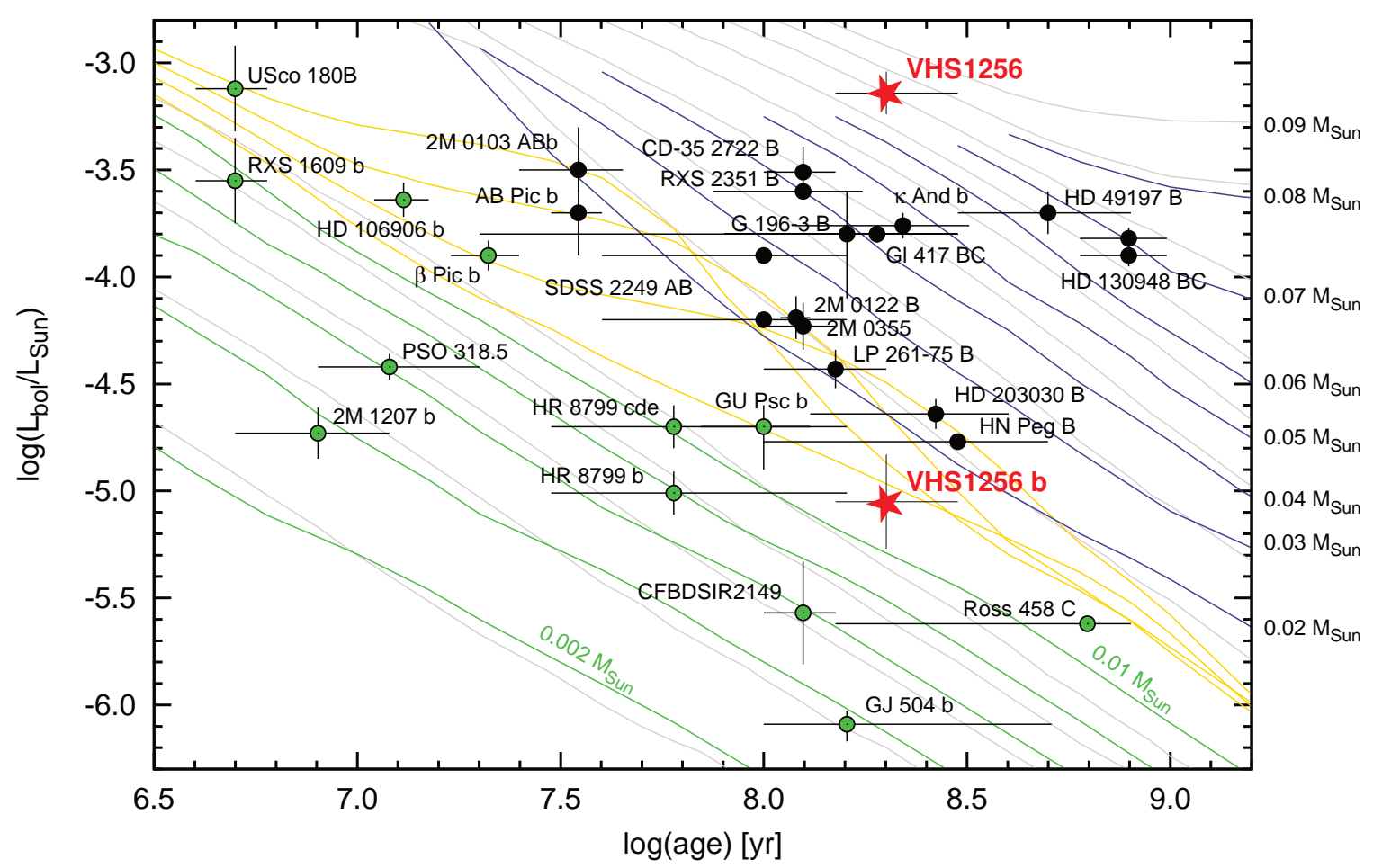

FIG. 13. - Luminosity and age of the two components of VHS 1256-1257 compared with evolutionary tracks from the solar abundance, cloudy atmosphere models of Saumon \& Marley (2008) with $f_{\text {sed }}=2$ and from the BT-Settl models of the Lyon group (Allard \& Barman 2004). The Saumon \& Marley (2008) models are plotted in color, with $0.002-0.010 M_{\odot}$ tracks in green (spaced by $\left.0.002 M_{\odot}\right), 0.011-$ $0.014 M_{\odot}$ tracks in yellow with a $0.001 M_{\odot}$ step, and $0.02-0.08 M_{\odot}$ in blue, with $0.01 M_{\odot}$ increments. The light gray curves correspond to BT-Settl models. For comparison purposes we overplotted the population of known young substellar objects, from the compilations by Bowler et al. (2013), Liu et al. (2013) and updated with recently discovered objects GU Psc b (Naud et al. 2014) and HD 106906 b (Bailey et al. 2014). The BT-Settl isomass lines yield slightly higher luminosities at masses $>0.030 M \odot$.

2013), HN Peg B (Luhman et al. 2007), 2MASS 1207 b (Skemer et al. 2011; Barman et al. 2011b). Moreover, at this temperature level of $\sim 900 \mathrm{~K}$ we would already expect to see the methane absorption bands in the near-IR spectra of the companion. The absence of methane was observed in other similar objects, like for example the HR 8799 planets. On the contrary, if the actual temperature is higher, like that corresponding to "normal" field L6-L8 dwarfs, this would lead to unrealistically small radius of the object $\left(\sim 0.5 R_{\mathrm{Jup}}\right)$, given its bolometric luminosity.

We compare in Figure 13 the luminosity of the companion at the adopted range of ages with the Saumon \& Marley (2008) and BT-Settl models (Allard \& Barman 2004) and with presently known young substellar objects, compiled by Bowler et al. (2013) updated with recently reported young T-dwarf companion GU PSc b (Naud et al. 2014) and a planetary mass companion HD 106906 b (Bailey et al. 2014). Interpretation of evolutionary tracks in this specific region has to be considered with caution. At that range of ages and luminosities the onset of deuterium burning causes overlapping of isomass tracks and less massive object can be slightly more luminous than a more massive one. This may introduce ambiguities in the determination of masses and produce a relatively large uncertainty in the upper limit of the derived mass of the companion. This object for its location in the HR diagram and its close distance is an ideal case for the application of the deuterium test (Béjar et al. 1999, Chabrier et al. 2000a).

\section{SUMMARY AND FINAL REMARKS}

We have identified an unusually red $\left(J-K_{s}=2.47\right.$ mag) common proper motion $\mathrm{L} 7 \pm 1.5$ type companion located at 8."06 \pm 0 ". $03(\sim 102 \mathrm{AU})$ of an M7.5 \pm 0.5 dwarf. The near-IR spectrum of the secondary shows a peaked triangular shape of the $H$-band continuum, and other spectral features recognized as hallmarks of low surface gravity and youth. The optical spectrum of the primary shows no $\mathrm{Li}$ I at $670.82 \mathrm{~nm}$ at a limit of $\mathrm{pEW}<30 \mathrm{~m} \AA$. We have determined a parallactic distance of the system of $12.7 \pm 1.0 \mathrm{pc}$, which is in agreement with the distance estimated from spectral type and photometry of the primary. From the proper motion, distance, and radial velocity of the VHS 1256-1257 we obtained the galactic velocities of the primary, which indicate that the system likely belongs to the Local Association. The non-detection of lithium and the kinematics of the primary allowed us to constrain the age of the system in the range of 150-300 Myr. From near-IR photometry and bolometric corrections we estimate luminosities of $\log \left(L_{\mathrm{bol}} / L_{\odot}\right)$ of $-3.14 \pm 0.10$ and $-5.05 \pm 0.22$ dex for the primary and secondary, respectively. By comparison with theoretical evolutionary models we derived a mass of $73_{-15}^{+20} M_{\text {Jup }}$ for the primary, at around the substellar mass limit and $11.2_{-1.8}^{+9.7} M_{\text {Jup }}$ for the secondary, near the deuterium-burning mass limit. At the distance of $12.7 \mathrm{pc}$ VHS $1256-1257 \mathrm{~b}$ is among the nearest currently known planetary mass companions detected by direct imaging. Moreover, it is one of the very few young, extremely red 
L dwarfs with age constrained within a narrow range, given by the likely belonging to the Local Association and the absence of Li I in the primary.

The $T_{\text {eff }}$ of $\sim 900 \mathrm{~K}$ determined from evolutionary models based on the luminosity does not seem to be consistent with the expected $T_{\text {eff }}$ range of field dwarfs of similar spectral type and with the absence of methane, which is expected to appear in atmosphere cooler than 1400 K. Following Barman et al. (2011a b) the formation of clouds with substantial vertical thickness and non-equilibrium chemistry in a low-gravity object like VHS 1256-1257 b could provide an explanation of the apparent high atmospheric temperature $(>1500 \mathrm{~K})$ as compared to cooling track effective temperature predictions (900-1000 K). As atmospheric clouds are composed mostly of $\mathrm{Fe}$ and $\mathrm{Mg}$-Si grains, we argue that the infall of planetesimals into this, and other young planetary mass objects, may enrich their atmospheres with key ingredients for substantial cloud formation. As time progresses, the amount of infalling material will decrease, the surface gravity of the planet will increase, and therefore the formation of thick clouds will become less important. As a consequence, the majority of field mid L dwarfs will not display the extreme IR colors found in these young objects. An alternative hypothesis to aid the explanation of the very red colors involves presence of a warm debris disk or a dust-shell surrounding the object and causing the extinction.

Since it is a relatively nearby and bright object near the deuterium-burning limit, it becomes one of the most promising targets to study the application of the deuterium test. From the masses and separation of the components we estimate the orbital period to be about 3900 yr. Assuming a circular orbit with a face-on orientation, the displacement caused by the orbital motion would be from 4 to 13 mas/yr, which will become feasible to measure in the next few years using precise astrometric observations.
We thank the anonymous referee for his/her valuable comments that improved this manuscript. B.G. would like to kindly thank Dr. Michael Liu for providing the spectra of PSO J318.5-22. We thank Roi Alonso and Aitor Bereciartua for performing the IAC80 observations used in this work. N.L. was funded by the Ramón y Cajal fellowship number 08-303-01-02. N.L. and V.J.S.B. are financially supported by the projects AYA2010-19136 and AYA2010-20535 from the Spanish Ministry of Economy and Competitiveness (MINECO), respectively. A.P.G. has been supported by Project No. 15345/PI/10 from the Fundación Séneca and MINECO under the grant AYA2011-29024. We thank the ESO staff for carrying out the VLT-UVES observations. Allocation of VLT Directors Discretionary Time is gratefully acknowledged. This work is based on observations collected at the European Southern Observatory, Chile, under program numbers 092.C-0874 and 293.C-5014(A). Based on observation obtained as part of the VISTA Hemisphere Survey, ESO progamme, 179.A-2010 (PI: McMahon). The VISTA Data Flow System pipeline processing and science archive are described in Irwin et al. (2004) and Cross et al. (2009). This work is based on observations (program GTC65-13B; PI Lodieu) made with the Gran Telescopio Canarias (GTC), operated on the island of La Palma in the Spanish Observatorio del Roque de los Muchachos of the Instituto de Astrofísica de Canarias. This article is based on observations made with IAC80, NOT, and WHT operated on the islands of Tenerife and La Palma by IAC, NOTSA and ING in the Spanish Observatorio del Teide and Roque de los Muchachos. The data presented here were obtained (in part) with ALFOSC, which is provided by the Instituto de Astrofísica de Andalucía (IAA) under a joint agreement with the University of Copenhagen and NOTSA. This research has benefitted from the Ultracool RIZzo Spectral Library (http://dx.doi.org/10.5281/zenodo.11313), maintained by Jonathan Gagné and Kelle Cruz.

\section{REFERENCES}

Ahn, C. P., Alexandroff, R., Allende Prieto, C., et al. 2012, ApJS, 203, 21

Allard, F., \& Barman, T. S. 2004, in IAU Symposium, Vol. 213 Bioastronomy 2002: Life Among the Stars, ed. R. Norris \& F. Stootman, 119

Allers, K. N., \& Liu, M. C. 2013, ApJ, 772, 79

Asiain, R., Figueras, F., Torra, J., \& Chen, B. 1999, A\&A, 344, 376

Bailey, V., Meshkat, T., Reiter, M., et al. 2014, ApJL, 780, L4

Baraffe, I., Chabrier, G., Allard, F., \& Hauschildt, P. H. 1998, A\&A, 337, 403

Baraffe, I., Chabrier, G., Barman, T. S., Allard, F., \& Hauschildt, P. H. 2003, A\&A, 402, 701

Barman, T. S., Macintosh, B., Konopacky, Q. M., \& Marois, C. 2011a, ApJ, 733, 65

- 2011b, ApJL, 735, L39

Basri, G., Marcy, G. W., \& Graham, J. R. 1996, ApJ, 458, 600

Béjar, V. J. S., Osorio, M. R. Z., \& Rebolo, R. 1999, ApJ, 521, 671

Béjar, V. J. S., Zapatero Osorio, M. R., Rebolo, R., et al. 2011, ApJ, 743, 64

Bihain, G., Rebolo, R., Béjar, V. J. S., et al. 2006, A\&A, 458, 805

Bihain, G., Rebolo, R., Zapatero Osorio, M. R., Béjar, V. J. S., \& Caballero, J. A. 2010, A\&A, 519, A93

Bonfils, X., Delfosse, X., Udry, S., et al. 2013, A\&A, 549, A109

Bonnefoy, M., Chauvin, G., Lagrange, A.-M., et al. 2014, A\&A, 562, A 127

Bowler, B. P., Liu, M. C., Shkolnik, E. L., \& Dupuy, T. J. 2013, ApJ, 774, 55

Burgasser, A. J., Cruz, K. L., Cushing, M., et al. 2010, ApJ, 710, 1142
Caffau, E., Ludwig, H.-G., Steffen, M., Freytag, B., \& Bonifacio, P. 2011, SoPh, 268, 255

Cepa, J. 2010, in Highlights of Spanish Astrophysics V, ed. J. M. Diego, L. J. Goicoechea, J. I. González-Serrano, \& J. Gorgas, 15 Chabrier, G., \& Baraffe, I. 1997, A\&A, 327, 1039

Chabrier, G., Baraffe, I., Allard, F., \& Hauschildt, P. 2000a, ApJL, 542, L119

-. 2000b, ApJ, 542, 464

Chauvin, G., Lagrange, A.-M., Dumas, C., et al. 2005, A\&A, 438, L25

Chauvin, G., Vigan, A., Bonnefoy, M., et al. 2014, ArXiv e-prints, arXiv:1405.1560

Chubak, C., Marcy, G., Fischer, D. A., et al. 2012, ArXiv e-prints, arXiv:1207.6212

Cross, N. J. G., Collins, R. S., Hambly, N. C., et al. 2009, MNRAS, 399, 1730

Cruz, K. L., Kirkpatrick, J. D., \& Burgasser, A. J. 2009, AJ, 137, 3345

Cruz, K. L., Reid, I. N., Liebert, J., Kirkpatrick, J. D., \& Lowrance, P. J. 2003, AJ, 126, 2421

Cruz, K. L., Reid, I. N., Kirkpatrick, J. D., et al. 2007, AJ, 133, 439

Cushing, M. C., Rayner, J. T., \& Vacca, W. D. 2005, ApJ, 623, 1115

Dahn, C. C., Harris, H. C., Vrba, F. J., et al. 2002, AJ, 124, 1170

Dalton, G. B., Caldwell, M., Ward, A. K., et al. 2006, in Society of Photo-Optical Instrumentation Engineers (SPIE) Conference Series, Vol. 6269, Society of Photo-Optical Instrumentation Engineers (SPIE) Conference Series

Deacon, N. R., \& Hambly, N. C. 2007, A\&A, 468, 163

Deacon, N. R., Liu, M. C., Magnier, E. A., et al. 2014, ApJ, 792, 119 
Dupuy, T. J., \& Liu, M. C. 2012, ApJS, 201, 19

Eggen, O. J. 1992, AJ, 104, 2141

Emerson, J. P. 2001, in Astronomical Society of the Pacific Conference Series, Vol. 232, The New Era of Wide Field Astronomy, ed. R. Clowes, A. Adamson, \& G. Bromage, 339

Emerson, J. P., Sutherland, W. J., McPherson, A. M., et al. 2004, The Messenger, 117, 27

Faherty, J. K., Burgasser, A. J., Cruz, K. L., et al. 2009, AJ, 137,

Faherty, J. K., Burgasser, A. J., West, A. A., et al. 2010, AJ, 139, 176

Faherty, J. K., Cruz, K. L., Rice, E. L., \& Riedel, A. 2013a, MmSAI, 84, 955

Faherty, J. K., Cruz, K. L., Rice, E. L., \& Riedel, A. 2014, in IAU Symposium, Vol. 299, IAU Symposium, ed. M. Booth, B. C. Matthews, \& J. R. Graham, 36-37

Faherty, J. K., Rice, E. L., Cruz, K. L., Mamajek, E. E., \& Núñez, A. 2013b, AJ, 145, 2

Faherty, J. K., Burgasser, A. J., Walter, F. M., et al. 2012, ApJ, 752,56

Freudling, W., Romaniello, M., Bramich, D. M., et al. 2013, A\&A, 559, A96

Fuhrmeister, B., Lalitha, S., Poppenhaeger, K., et al. 2011, A\&A, 534, A133

Gagné, J., Lafrenière, D., Doyon, R., et al. 2014, ApJL, 792, L17

Gálvez-Ortiz, M. C., Kuznetsov, M., \& et al. 2014, MNRAS, 439, 3890

Golimowski, D. A., Leggett, S. K., Marley, M. S., et al. 2004, AJ, 127,3516

Gorlova, N. I., Meyer, M. R., Rieke, G. H., \& Liebert, J. 2003, ApJ, 593, 1074

Green, R. M. 1985, Spherical astronomy

Griffin, R., \& Griffin, R. 1973, MNRAS, 162, 255

Henry, T. J., \& Kirkpatrick, J. D. 1990, ApJL, 354, L29

Høg, E., Kuzmin, A., Bastian, U., et al. 1998, A\&A, 335, L65

Høg, E., Fabricius, C., Makarov, V. V., et al. 2000, A\&A, 355, L27

Irwin, M. J., Lewis, J., Hodgkin, S., et al. 2004, in Society of Photo-Optical Instrumentation Engineers (SPIE) Conference Series, Vol. 5493, Optimizing Scientific Return for Astronomy through Information Technologies, ed. P. J. Quinn \& A. Bridger, 411-422

Jameson, R. F., Lodieu, N., Casewell, S. L., Bannister, N. P., \& Dobbie, P. D. 2008, MNRAS, 385, 1771

Johnson, D. R. H., \& Soderblom, D. R. 1987, AJ, 93, 864

Johnson, H. L. 1966, ARA\&A, 4, 193

Kausch, W., Noll, S., Smette, A., et al. 2015, A\&A, 576, A78

Kirkpatrick, J. D., Barman, T. S., Burgasser, A. J., et al. 2006, ApJ, 639, 1120

Kirkpatrick, J. D., Beichman, C. A., \& Skrutskie, M. F. 1997, ApJ, 476, 311

Kirkpatrick, J. D., Henry, T. J., \& McCarthy, Jr., D. W. 1991, ApJS, 77, 417

Kirkpatrick, J. D., Kelly, D. M., Rieke, G. H., et al. 1993, ApJ, 402,643

Knapp, G. R., Leggett, S. K., Fan, X., et al. 2004, AJ, 127, 3553

Kuzuhara, M., Tamura, M., Kudo, T., et al. 2013, ApJ, 774, 11

Lagrange, A.-M., Gratadour, D., Chauvin, G., et al. 2009, A\&A, 493, L21

Landolt, A. U. 1992, AJ, 104, 340

-. 2009, AJ, 137, 4186

Leggett, S. K., Allard, F., Dahn, C., et al. 2000, ApJ, 535, 965

Lewis, J. R., Irwin, M., \& Bunclark, P. 2010, in Astronomical Society of the Pacific Conference Series, Vol. 434, Astronomical Data Analysis Software and Systems XIX, ed. Y. Mizumoto, K.-I. Morita, \& M. Ohishi, 91

Liu, M. C., Magnier, E. A., Deacon, N. R., et al. 2013, ApJL, 777, L20
Luhman, K. L. 2012, ARA\&A, 50, 65

-. 2013, ApJL, 767, L1

Luhman, K. L., Patten, B. M., \& et al. 2007, ApJ, 654, 570

Marois, C., Macintosh, B., Barman, T., et al. 2008, Science, 322, 1348

Marois, C., Zuckerman, B., Konopacky, Q. M., Macintosh, B., \& Barman, T. 2010, Nature, 468, 1080

Martín, E. L., Basri, G., Gallegos, J. E., et al. 1998, ApJL, 499, L61

McLean, I. S., McGovern, M. R., Burgasser, A. J., et al. 2003,

ApJ, 596, 561

McMahon, R. G., Banerji, M., Gonzalez, E., et al. 2013, The Messenger, 154, 35

Melis, C., Reid, M. J., Mioduszewski, A. J., Stauffer, J. R., \&

Bower, G. C. 2014, Science, 345, 1029

Naud, M.-E., Artigau, É., Malo, L., et al. 2014, ApJ, 787, 5

Nidever, D. L., Marcy, G. W., Butler, R. P., Fischer, D. A., \&

Vogt, S. S. 2002, ApJS, 141, 503

Pancino, E., Altavilla, G., Marinoni, S., et al. 2012, MNRAS, 426, 1767

Patience, J., King, R. R., de Rosa, R. J., \& Marois, C. 2010, A\&A, 517, A76

Peña Ramírez, K., Béjar, V. J. S., Zapatero Osorio, M. R.,

Petr-Gotzens, M. G., \& Martín, E. L. 2012, ApJ, 754, 30

Pinfield, D. J., Jones, H. R. A., Lucas, P. W., et al. 2006, MNRAS, 368, 1281

Rayner, J. T., Cushing, M. C., \& Vacca, W. D. 2009, ApJS, 185, 289

Rebolo, R., Martin, E. L., Basri, G., Marcy, G. W., \&

Zapatero-Osorio, M. R. 1996, ApJL, 469, L53

Reid, I. N., Cruz, K. L., Kirkpatrick, J. D., et al. 2008, AJ, 136, 1290

Reid, I. N., \& Hawley, S. L. 1999, AJ, 117, 343

Reiners, A., \& Basri, G. 2010, ApJ, 710, 924

Saumon, D., \& Marley, M. S. 2008, ApJ, 689, 1327

Skemer, A. J., Close, L. M., Szücs, L., et al. 2011, ApJ, 732, 107

Skrutskie, M. F., Cutri, R. M., Stiening, R., et al. 2006, AJ, 131, 1163

Smette, A., Sana, H., Noll, S., et al. 2015, A\&A, 576, A77

Soderblom, D. R., Nelan, E., Benedict, G. F., et al. 2005, AJ, 129,1616

Stauffer, J. R., Schultz, G., \& Kirkpatrick, J. D. 1998, ApJL, 499, L199

Torres, C. A. O., Quast, G. R., Melo, C. H. F., \& Sterzik, M. F. 2008, Young Nearby Loose Associations, ed. B. Reipurth, 757

Ushomirsky, G., Matzner, C. D., Brown, E. F., et al. 1998, ApJ, 497,253

van Leeuwen, F. 2007, A\&A, 474, 653

Vrba, F. J., Henden, À. A., Luginbuhl, C. B., et al. 2004, AJ, 127, 2948

Wahhaj, Z., Liu, M. C., Biller, B. A., et al. 2011, ApJ, 729, 139

West, A. A., Morgan, D. P., \& et al. 2011, AJ, 141, 97

Wright, E. L., Eisenhardt, P. R. M., \& et al. 2010, AJ, 140, 1868

Zapatero Osorio, M. R., Béjar, V. J. S., Miles-Páez, P. A., et al. 2014a, A\&A, 568, A6

Zapatero Osorio, M. R., Béjar, V. J. S., Pavlenko, Y., et al. 2002, A\&A, 384, 937

Zapatero Osorio, M. R., Gálvez Ortiz, M. C., Bihain, G., et al. 2014b, A\&A, 568, A77

Zapatero Osorio, M. R., Béjar, V. J. S., Martín, E. L., et al.

2014c, ArXiv e-prints, arXiv:1410.2383

Zuckerman, B., \& Song, I. 2004, ARA\&A, 42, 685 